Draft version September 17, 2018

Preprint typeset using $\mathrm{LAT}_{\mathrm{E}} \mathrm{X}$ style emulateapj v. 5/2/11

\title{
HERSCHEL AND SPITZER OBSERVATIONS OF SLOWLY ROTATING, NEARBY ISOLATED NEUTRON STARS
}

\author{
B. Posselt \\ Department of Astronomy \& Astrophysics, Pennsylvania State University, 525 Davey Lab,University Park, PA 16802, USA \\ G. G. PAVLOV \\ Department of Astronomy \& Astrophysics, Pennsylvania State University, 525 Davey Lab,University Park, PA 16802, USA \\ S. POPOV \\ Sternberg Astronomical Institute, Lomonosov Moscow State University, Moscow 119992, Russia \\ S. WACHTER \\ Max Planck Institute for Astronomy, Königsstuhl 17, 69117 Heidelberg, Germany \\ Draft version September 17, 2018
}

\begin{abstract}
Supernova fallback disks around neutron stars have been discussed to influence the evolution of the diverse neutron star populations. Slowly rotating neutron stars are most promising to find such disks. Searching for the cold and warm debris of old fallback disks, we carried out Herschel PACS $(70 \mu \mathrm{m}$, $160 \mu \mathrm{m})$ and Spitzer IRAC $(3.6 \mu \mathrm{m}, 4.5 \mu \mathrm{m})$ observations of eight slowly rotating $(P \approx 3-11 \mathrm{~s})$ nearby $(<1 \mathrm{kpc})$ isolated neutron stars. Herschel detected $160 \mu \mathrm{m}$ emission $(>5 \sigma)$ at locations consistent with the positions of the neutron stars RX J0806.4-4123 and RX J2143.0+0654. No other significant infrared emission was detected from the eight neutron stars. We estimate probabilities of $63 \%, 33 \%$ and $3 \%$ that, respectively, none, one, or both Herschel PACS $160 \mu \mathrm{m}$ detections are unrelated excess sources due to background source confusion or an interstellar cirrus. If the $160 \mu \mathrm{m}$ emission is indeed related to cold ( $10 \mathrm{~K}$ to $22 \mathrm{~K}$ ) dust around the neutron stars, this dust is absorbing and re-emitting $\sim 10 \%$ to $\sim 20 \%$ of the neutron stars' X-rays. Such high efficiencies would be at least three orders of magnitude larger than the efficiencies of debris disks around nondegenerate stars. While thin dusty disks around the neutron stars can be excluded as counterparts of the $160 \mu \mathrm{m}$ emission, dusty asteroid belts constitute a viable option.

Subject headings: pulsars: individual (RX J0420.0-5022, RX J0720.4-3125, RX J0806.44123，RX J1308.6+2127，RXJ1605.3+3249，RX J1856.5-3754，PSR J1848-1952, RX J2143.0+0654 ) — stars: neutron
\end{abstract}

\section{INTRODUCTION}

During the last decade sensitive X-ray observations have revealed a rather surprising diversity of neutron stars (NSs); for a review see, e.g., Kaspi (2010). While most of the currently known $\sim 2000$ NSs are rotationpowered radio pulsars, there are also rotating radio transients, central compact objects (CCOs) in supernova remnants, X-ray thermal isolated NSs (XTINSs) and magnetars. The group of the magnetars, consisting of the Anomalous X-ray Pulsars (AXPs) and the Soft Gamma-ray Repeaters (SGRs), posed particular challenges to the simplistic NS model as a rotating magnetic dipole which converts rotational energy into electromagnetic pulsar emission. Magnetar quiescent emission and the occasionally observed huge magnetar flares require an additional energy source. The prevailing model for magnetars is that of young, isolated NSs powered by decay of very large magnetic fields (Duncan \& Thompson 1992). Within this model the flares are understood as reconnection events in a twisted magnetosphere (e.g., Thompson et al. 2002). In addition to the dipole

posselt@psu.edu magnetic fields, recent magneto-thermal NS evolution models consider crustal toroidal magnetic fields as well (e.g., Pons \& Geppert 2007; Pons et al. 2009). These models have been successful in explaining, for example, the observed numbers of magnetars, XTINSs and radio pulsars (Popov et al. 2010), or the observed properties of CCOs (Viganò \& Pons 2012).

However, there are viable additional or alternative factors which may influence the evolution and observed diversity of NSs. Different masses of supernova fallback disks could exert different disk torques, leading to different accretion rates and ultimately to the diversity of observed NS classes (e.g., Alpar 2001; Alpar et al. 2001). Explaining the emission of magnetars as the most extreme case has become the litmus test for this theory. Accretion disks around magnetars have initially been suggested and discussed by van Paradijs et al. (1995) and Chatterjee et al. (2000). The theory was supported by recent works (e.g., Trümper et al. 2013; Ertan \& Calıskan 2006) which explain the X-ray spectral and timing properties, as well as the radio, optical and IR properties of magnetars. Note, however, that these mod- 
els cannot produce the observed giant magnetar flares which are attributed to local ultra-strong multipole fields in an analogy to star spots (Trümper et al. 2013). Recently, Caliskan et al. (2013) expanded the application of the fallback model to a different NS class, represented by radio pulsars with high magnetic fields. These recent results imply that fallback disks currently cannot be excluded as crucial ingredients in the NS evolution. In fact, such disks are a general prediction of supernova models (Michel \& Dessler 1981; Chevalier 1989), however, observationally they have remained rather elusive; for a review about searches for disks around NSs see Wang (2014). There is so far only one example of a likely fallback disk

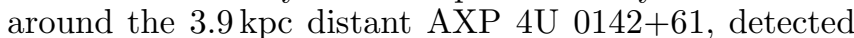
with Spitzer IRAC (Wang et al.|2006; Ertan et al.|2007). According to Ekssi \& Alpar (2005), fallback disks are expected to be rare because they are likely to be disrupted when the newly born NS spins rapidly through the propeller stage, at which inflowing matter would be expelled instead of being accreted. The fallback disks can survive if the initial NS spin is slow enough $\left(P_{0} \geq 40 \mathrm{~ms}\right.$ at a magnetic moment of $\left.\mu=10^{30} \mathrm{G} \mathrm{cm}^{3}\right)$. The presence of disks around highly magnetized NSs today is therefore, in principle, related to the initial birth parameters of these NSs, in particular, to the origin of the strong fields in highly magnetized NSs. It is important to obtain more comprehensive observational constraints on the presence of supernova fallback disks around the different NS classes. Slowly rotating NSs, such as the magnetars and the XTINS, appear to be most promising to find such disks.

\section{SAMPLE SELECTION AND WORKING HYPOTHESES}

In the framework of the fallback disk model, Alpar (2001, 2007) proposed that different initial disk masses lead to the different NS classes. In this picture, magnetars are expected to have the most massive disks, followed by those of the XTINS, while rotation powered radio pulsars have negligible or no disks. Trümper et al. (2010) suggested that XTINSs may be more evolved versions of AXPs with fallback disks. It is interesting to note that in the $P-\dot{P}$ diagram the XTINS are located together with a few high- $B$ pulsars between the separated groups of magnetars and radio pulsars (see, e.g., Figure 2 in Kaspi 2010). Thus, these NSs may constitute a key population to unify the different NS classes.

Of the 26 currently known magnetars and magnetar candidates only 5 have been investigated at infrared wavelengths (Olausen \& Kaspi 2013). Apart from the detections of AXP 4U 0142+61 (Wang et al. 2006) and 1E 2259+586 (Kaplan et al. 2009), the shallow mid-IR Spitzer searches of three other magnetars were not very constraining (Wang et al. 2007b). It is difficult to constrain the disk emission around magnetars because most of these NSs are far away. The median distance of magnetars with known distances is $8.5 \mathrm{kpc}$ (Olausen \& Kaspi 2013)]. The XTINS, however, are all expected to be within $1 \mathrm{kpc}$ based on parallaxes and the interstellar absorption of their soft X-ray spectra (e.g., Kaplan et al. 2009; Posselt et al. 2007). Such distances

1 McGill SGR/AXP Online Catalog (March 2014): www.physics.mcgill.ca/p̃ulsar/magnetar/main.html enable constraining searches for fallback disks for the whole known XTINS population. We selected all known slowly rotating $(P>3 \mathrm{~s}) \mathrm{NSs}$ within $1 \mathrm{kpc}$. Our sample includes all the seven known XTINS and the nearby high $-B$ radio pulsar PSR J1848-1952 which has similar spin-down properties (e.g., Kaplan et al. 2009).

In the framework of the fallback disk model, potential XTINS disks have likely smaller masses than magnetar disks, are likely older than those, and are likely passive, i.e., without significant on-going accretion on the NS. The latter property can be concluded from the very stable X-ray emission of the XTINSs with the notable exception of RX J0720.4-3125 (e.g., Haberl 2007; van Kerkwijk et al. 2007; Hohle et al. 2012).

The composition and evolution of fallback disks are unknown. Dust grain evolution was shown to decouple from gas evolution in nearly all (protoplanetary) disk configurations if the initial dust-to-gas ratio is larger than 0.03 (Pinte \& Laibe 2014). Given the supernova origin, one can reasonably assume metal-rich disk material, i.e., a decoupled dust evolution in the disk. Gas is easier removed from the disks than dust grains by the pulsar wind and the interstellar ram pressure. We will therefore assume dust-rich disks as a working hypothesis for potential residual XTINS fallback disks. In comparison to other stars, the potential XTINS disks may more likely resemble transitional or debris disks such as those around main-sequence stars (e.g., Wyatt 2008 and references therein), central stars in planetary nebulae (e.g., Su et al. 2007), or white dwarfs (e.g, Debes et al. 2012 and references therein) - than protostellar/protoplanetary disks around young stars.

\section{OBSERVATIONS}

\subsection{Herschel Observations}

Herschel observations of eight isolated NSs were carried out over a period of $\sim 1$ year using the Photodetector Array Camera and Spectrometer (PACS, Poglitsch et al. 2010). We obtained the images in mini-map mode in the blue $(60-85 \mu \mathrm{m})$ and red $(130-210 \mu \mathrm{m})$ bands with the respective PACS bolometer arrays operated in parallel. The mini-map mode is a particular case of the scanmap mode, where two observations (scan and cross-scan) are carried out which have scan angles in array coordinates along the two array diagonal directions, 70 and 110 degrees. An individual scan-map observation has a pattern of parallel, overlapping scan lines connected by turnaround loops. The mini-map mode provides a good point source sensitivity with a relatively large homogeneous coverage (about $50^{\prime \prime}$ in diameter) 2 . Our observations were done with medium scan speed $\left(20^{\prime \prime} \mathrm{s}^{-1}\right)$, the mapping parameters were 10 scan legs with scan lengths of $3^{\prime}$ with a separation of $4^{\prime \prime}$ between them. A repetition factor of 12 was chosen to reach point source sensitivities close to the expected confusion noise (for a details about the confusion noise in the PACS bands and its estimation see, e.g., the Herschel Observers' Manua[3, Chapter 4.3.

2 PACS Observer's Manual; Chapter 5.2; herschel.esac.esa.int/Docs/PACS/html/pacs_om.html

${ }^{3}$ herschel.esac.esa.int/Docs/Herschel/html/Observatory.html 
Table 1

List of Herschel observations

\begin{tabular}{lccc}
\hline \hline \multicolumn{1}{c}{ Object } & Date & ObsIDs & PointAcc $^{\mathrm{a}}$ \\
\hline RX J0420.0-5022 & $2012-01-07$ & $1342236938 / 9$ & $1^{\prime \prime} 1$ \\
RX J0720.4-3125 & $2011-11-10$ & $1342232218 / 9$ & $1^{\prime \prime} 1$ \\
RX J0806.4-4123 & $2011-10-29$ & $1342231572 / 3$ & $1^{\prime \prime} 1$ \\
RX J1308.6+2127 & $2011-07-11$ & $1342223951 / 2$ & $1^{\prime \prime} 5$ \\
RX J1605.3+3249 & $2011-07-20$ & $1342224486 / 7$ & $1^{\prime \prime} 5$ \\
RX J1856.5-3754 & $2011-03-15$ & $1342216058 / 9$ & $2^{\prime \prime} 4$ \\
PSR J1848-1952 & $2012-03-13$ & $1342241353 / 4$ & $1^{\prime \prime} 1$ \\
RX J2143.0+0654 & $2012-04-23$ & $1342244870 / 1$ & $1^{\prime \prime} 1$ \\
\hline
\end{tabular}

a $1 \sigma$ absolute pointing accuracy; see text in Section 4.1 .

and the HERSCHEL-HSC-DOC-0886 document by C. Kiss). Our observations are listed in Table 1. Overall, each Astronomical Observation Request (AOR) was $7 \mathrm{ks}$. The beam sizes (full width of half maximum, FWHM) of the blue and red band at medium speed are 5." $5 \times 5$ ". 8 and $10^{\prime \prime} .5 \times 12$.' 02 , respectively (Poglitsch et al. 2010).

\subsection{Spitzer Observations}

We observed RX J0720.4-3125 with the Spitzer Space Telescopt5 (Werner et al. 2004) during the postcryogenic mission in January 2011 (PI: Posselt). The observations were done with the Infrared Array Camera (IRAC; Fazio et al. 2004) at $3.6 \mu \mathrm{m}$ and $4.5 \mu \mathrm{m}$. We used a frame time of $100 \mathrm{~s}$ and 31 dither positions in each band. We also consider here some of the NS observations of another Spitzer program (PI: Wachter) which searched for fallback disks around a large number of nearby pulsars using the IRAC $4.5 \mu \mathrm{m}$ channel. Those observations were done from September 2011 to December 2012. In each observation a 100 s frame time and 30 dither positions were used. The Spitzer observations discussed in this paper are listed in Table 2 The IRAC field of view is $5 ! 2 \times 5 ! 2$, the native IRAC pixel size is $1^{\prime \prime} .2$, the FWHM of point spread function at $3.6 \mu \mathrm{m}$ and $4.5 \mu \mathrm{m}$ is $1^{\prime \prime} 66$ and $1^{\prime \prime} .72$, respectively (Fazio et al. 2004).

Table 2

List of Spitzer observations

\begin{tabular}{lccc}
\hline Object & Date & Channels & AOR \\
\hline RX J0420 & $2011-09-17$ & $4.5 \mu \mathrm{m}$ & 42478336 \\
RX J0720 & $2011-11-21$ & $3.6 \& 4.5 \mu \mathrm{m}$ & 39947264 \\
RX J0806 & $2012-04-30$ & $4.5 \mu \mathrm{m}$ & 42478592 \\
RX J1308 & $2011-07-24$ & $4.5 \mu \mathrm{m}$ & 42478848 \\
RX J1605 & $2012-05-01$ & $4.5 \mu \mathrm{m}$ & 42479616 \\
RX J1856 & $2011-11-12$ & $4.5 \mu \mathrm{m}$ & 42479360 \\
RX J2143 & $2012-12-31$ & $4.5 \mu \mathrm{m}$ & 42479872 \\
\hline
\end{tabular}

\section{DATA REDUCTION AND FLUX MEASUREMENT PROCEDURE}

\subsection{Herschel Observations}

Using the Herschel Interactive Processing Environment (HIPE), v9.06, we reduced the data based on the deep-survey scan-map pipeline scripts of the PACS

\footnotetext{
${ }^{4}$ herschel.esac.esa.int/Docs/HCNE/pdf/HCNE_ScienceDoc.pdf

5 irsa.ipac.caltech.edu/data/SPITZER/docs/spitzermission/

6 herschel.esac.esa.int/HIPE_download.shtml
}

photometer pipeline. The PACS calibration file set PACS_CAL_41_[7 was applied. We reprocessed the data from the Level 0 to the final maps (Level 2.5) applying the usual processing steps outlined in Poglitsch et al. (2010) and Lutz et al. (2011).

A critical step in the data processing is the removal of the $1 / \mathrm{f}$ noise, the heavy flicker noise of the PACS readout system. In the case of a point source observation, a high pass filter along the time line of the observation allows one to remove most of the $1 / \mathrm{f}$ noise, which increases the sensitivity of the observation. The effect of the high-pass filter on the PACS point spread function and the noise, the resulting flux removal and mask strategies to avoid the former are discussed in detail by Popesso et al. (2012). According to this study, the high-pass filter most efficiently removes noise in case of high data redundancy, i.e., large repetition factors. Flux losses of point sources are reduced best by putting circular mask patches on prior source positions. The filter width and patch sizes need to be carefully chosen since their influences dominate the global and local noise in the PACS maps. In our data processing we checked maps produced with a masking technique where only sources above a certain threshold are masked before the combined map is produced. This method can be expected to have the highest sensitivity for faint point source identification. However, not all observations show a PACS source near the isolated neutron star (INS) position. Aiming to reduce flux losses in our flux (limit) estimates, we therefore masked a region with radius $10^{\prime \prime}$ around each expected INS position for our final map productions.

The pixel noise of individual pixels in a PACS photometer map is correlated due to the $1 / \mathrm{f}$ noise and due to the applied drizzle projection method by Fruchter \& Hook (2002) during the map creation process. Popesso et al. (2012) calculated the individual noise contributions in dependence of the high-pass filter width, the output pixel size and the pixel drop fraction used in the drizzle method. In general, the noise level is lower in the high data redundancy case compared to the low data redundancy case, and smaller pixel sizes and smaller pixel drop fractions can be chosen in the former case to achieve the same noise limit. We checked different sets of parameters. As a good final choice for all our maps, we used pixel sizes of $1^{\prime \prime}$ in the blue band, $2^{\prime \prime}$ in the red band, a pixel drop fraction of 0.04 in both bands and high-pass filter widths of 15 readouts in the blue band and 25 readouts in the red band.

The expected positions of the INSs at the time of the Herschel observations were calculated from positions and proper motions (if known) in the literature, and they are listed in Table 3. Position errors are usually below $1^{\prime \prime}$. We comment on individual INS uncertainties in Section 5 and Appendix $\mathrm{A}$ if there are close neighbor sources in the Herschel images. The Herschel absolute pointing error ranges from $1 \sigma \approx 2$ '. 4 for our earliest Herschel observation (RX J1856) to $1 \sigma \approx 1^{\prime \prime} 1$ for our latest Herschel observations according to the Herschel

\footnotetext{
v41.html
} 
calibration and HIPE teams 8 . The pointing errors are listed in Table 1 .

We use apertures with sky annuli to measure source fluxes and apply the corresponding aperture corrections using the respective HIPE tasks. The calibration study by Popesso et al. (2012) provided a method to calculate aperture flux errors from the coverage of individual map pixels by considering the chosen data reduction parameters. First, the coverage-error map relation as well as the cross-correlation factor are calculated in dependence on the chosen pixel size, pixel drop fraction and high-pass filter width. Then, the coverage is used to calculate the error of the individual map pixels. The pixel errors are quadratically added for the respective aperture size. Finally, the cross-correlation factor is applied. This cross-correlation correction factor accounts for both components in the correlation noise, the projection itself and the residual $1 / f$ noise not removed by the high-pass filter. Since we give aperture-corrected fluxes, we apply the aperture correction factor to the estimated error as well. We checked whether the determined final error was of the same order as the variance of background apertures in nearby source-free regions and found this to be the case. We list $5 \sigma$ errors for the aperture-corrected fluxes in Table 5

If there is no source at the INS position, we consider several apertures to measure the standard deviation of the background in the central, source-free region of the map (close to the INS) where the exposure coverage is reasonably homogeneous. Depending on how crowded the field is, we usually obtain results from 7 to 15 apertures with radii of $5^{\prime \prime}$ or $7^{\prime \prime}$. We list $5 \sigma$ values for the flux limits in Table 5 .

We neglect the PACS photometer color corrections (for estimates of the monochromatic flux densities for different spectral shapes) because they are close to one for a wide range of expected disk temperatures ( $30 \mathrm{~K}$ to $1000 \mathrm{~K})$. However, if highly accurate fluxes for a particular disk model are desired, we refer to the respective Herschel PACS Technical Note PICC-ME-TN-0389.

\subsection{Spitzer Observations}

For the data reduction of the Spitzer IRAC data, we used Spitzer's MOsaicker and Point source Extractor package, MOPEX 10 , v.18.5.6. In the following we give an exemplary description of the data reduction for the IRAC data of RX J0720.4-3125 (two bands). The data reduction for the $4.5 \mu \mathrm{m}$ data of the other INSs is done following the same procedures.

In each case, we start from the artifact-corrected basic calibrated data (cBCD) frames. We removed one cBCD frame from the $3.6 \mu \mathrm{m}$ data set of RX J0720.4-3125 because it had a bad column at the target position. From the respective $4.5 \mu \mathrm{m}$ data set we removed 5 frames due

\footnotetext{
${ }^{8}$ herschel.esac.esa.int/twiki/bin/view/Public/SummaryPointing

9 herschel.esac.esa.int/twiki/pub/Public/PacsCalibrationWeb /cc_report_v1.pdf

10 irsa.ipac.caltech.edu/data/SPITZER/docs/dataanalysistools /tools/mopex/
}

to artifacts, possibly the so-called column pull-up, at or close to the source position. Using the mosaic task we obtained a combined image with a pixel size of 0.6 . We checked the alignment of the IRAC mosaic astrometry with the 2MASS Point Source Catalog (Skrutskie et al. 2006) using 114 2MASS sources with the highest quality flag AAA in the field. The astrometric accuracy of the $3.6 \mu \mathrm{m}$ and the $4.5 \mu \mathrm{m}$ mosaics of RX J0720.4-3125 are $0^{\prime \prime} .27$ and 0.28 , respectively. Similar astrometric accuracies are found for the other INS Spitzer mosaics. The expected INS positions were calculated for the times of the respective Spitzer observations using known positions and proper motions as described for Herschel in Section 4.1 .

The IRAC Point Response Function (PRF) is essentially the convolution of a box, having the size of the image pixel, with the point spread function (e.g., MOPEX user guide, chapter 8.9M. To consider a PRF which is variable in the field of view, one uses the so-called PRF maps which are provided by the Spitzer Science Center for the IRAC channels. The PRF is required for point source fitting photometry, which we performed with the apex multi-frame task on each individual $\mathrm{cBCD}$ frame. Applying then the apex-qa task, we subtracted the fitted point sources from the individual $\mathrm{cBCD}$ frame to obtain a residual mosaic. Such residual mosaics are useful if neighboring sources contaminate the flux at the source position.

We use aperture photometry to determine IRAC fluxes and flux limits. We apply aperture radii of 2 native IRAC pixels, corresponding to 4 mosaic pixels, and aperture correction factors of 1.21 and 1.22 for the $3.6 \mu \mathrm{m}$ and the $4.5 \mu \mathrm{m}$ measurements, respectively (Reach et al. 2005). To obtain an upper limit, we determine not only the source aperture flux, $F_{\mathrm{SRC}}$, but also the background level, $F_{\mathrm{BG}}$, and the standard deviation, $\sigma$, of several (usually 8-10) source-free apertures close to the source position. If necessary and possible, we use the residual mosaics for these measurements. In the case of a non-detection, we define the upper flux limit $F_{5 \sigma}=F_{\mathrm{SRC}}-F_{\mathrm{BG}}+5 \sigma$.

The derived IRAC fluxes are in Jy and correspond nominally to those fluxes one expects to measure in the respective IRAC channel for a source with $F_{\nu} \propto \nu^{-1}$. Sources with different spectral shapes, and in particular, very red sources require a color correction. The IRAC Instrument Handbook[3] (section 4.4) lists color corrections for different power law indices and blackbody temperatures. In Sections 5.1, 5.2 and A.1 to A.6, we give the aperture-corrected flux values without color correction, while in Table 5 we exemplarily apply the color corrections for a $T=200 \mathrm{~K}$ blackbody as an approximation for the spectral shape of potential warm disk emission.

\footnotetext{
11 irsa.ipac.caltech.edu/data/SPITZER/docs/dataanalysistools /tools/mopex/mopexusersguide/89/\#_Toc320000083

12 irsa.ipac.caltech.edu/data/SPITZER/docs/irac /calibrationfiles/psfprf/

13 irsa.ipac.caltech.edu/data/SPITZER/docs/irac /iracinstrumenthandbook/18/
} 
Table 3

Positions and proper motions of the eight neutron stars

\begin{tabular}{|c|c|c|c|c|c|c|c|c|c|}
\hline Object & $\begin{array}{l}\text { R.A. } \\
\text { h m s }\end{array}$ & $\begin{array}{l}\text { DEC } \\
\text { d m s }\end{array}$ & $\underset{\prime \prime}{\operatorname{PosErr}}{ }^{\mathrm{a}}$ & $\begin{array}{l}\text { Epoch } \\
\text { MJD }\end{array}$ & $\underset{c}{\text { Ref. }}$ & $\begin{array}{l}\mu_{\alpha} \cos \delta^{\mathrm{a}} \\
\operatorname{mas~yr}^{-1}\end{array}$ & $\begin{array}{c}\mu_{\delta}^{\mathrm{a}} \\
\text { mas } \mathrm{yr}^{-1}\end{array}$ & $\underset{c}{\operatorname{Ref} .}$ & $\begin{array}{c}\text { PosErrH } \\
\prime \prime(90 \%)\end{array}$ \\
\hline RX J0420 & 042001.94 & -502247.8 & $1.1(90 \%)$ & 54122 & 1 & \multicolumn{2}{|c|}{$\mu<123(2 \sigma)$} & 2 & 1.2 \\
\hline RX J0720 & 072024.93 & -312549.78 & $0.02,0.02(1 \sigma)$ & 54477 & 3 & $-93 \pm 5(1 \sigma)$ & $49 \pm 5(1 \sigma)$ & 3 & 0.06 \\
\hline RX J0806 & 080623.40 & -412230.9 & $0.6(90 \%)$ & 52326 & 4 & \multicolumn{2}{|c|}{$\mu<86(2 \sigma)$} & 2 & 0.9 \\
\hline RX J1308 & $1308 \quad 48.27$ & +212706.8 & $0.6(90 \%)$ & 51719 & 5 & $-207 \pm 20(2 \sigma)$ & $84 \pm 20(2 \sigma)$ & 2 & 0.7 \\
\hline RX J1605 & 160518.52 & +324918.0 & $0.3,0.3(1 \sigma)$ & 52111 & 6 & $25 \pm 16(90 \%)$ & $142 \pm 15(90 \%)$ & 7 & 0.7 \\
\hline RX J1856 & 185635.795 & -375435.54 & $0.1(90 \%)^{\mathrm{d}^{\prime}}$ & 52868 & 8 & $326.6 \pm 0.5(1 \sigma)$ & $-61.9 \pm 0.4(1 \sigma)$ & 8 & 0.1 \\
\hline PSR J1848 & 184818.04 & -195231 & $0.6,7(2 \sigma)$ & 48695 & 9 & $39 \pm 157(2 \sigma)$ & $-1200 \pm 1900(2 \sigma)$ & 9 & $3.1,9^{\mathrm{e}}$ \\
\hline RX J2143 & 214303.4 & +065417.5 & $0.2,0.2(1 \sigma)$ & 54388 & 10 & \multicolumn{2}{|c|}{$\mu<700(90 \%)$} & $t w^{f}$ & 3.2 \\
\hline
\end{tabular}

${ }^{a}$ Uncertainty levels as quoted in the respective papers. One value instead of two indicates the radial error.

b Positional radial error (90\% confidence level) of the NS position at the epoch of the respective Herschel observation, calculated using the Rayleigh distribution. In the case of different (position, proper motion) error values in R.A. and DEC we use the larger error for our estimate.

c References: 1 - Mignani et al. (2009), 2 - Motch et al. (2009), 3 - Eisenbeiss et al. (2010), 4 - Haberl et al. (2004), 5 - Kaplan et al. (2002), 6 - Kaplan et al. (2003), 7 - Motch et al. (2005), 8 - Walter et al. (2010), 9 - Hobbs et al. (2004), 10 - Schwope et al. (2009).

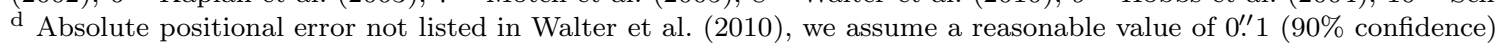

e Individual errors in R.A. and DEC, see discussion in Section A.6

f This work, see Section 5.2

\subsection{Complementary WISE data}

The Wide-field Infrared Survey Explorer (WISE, Wright et al. 2010) mapped the sky in 2010 at 3.4 (W1), 4.6(W2), 12(W3), and 22 (W4) $\mu \mathrm{m}$ with an angular resolution of $6 . " 1,6 . " 4,66^{\prime \prime} 5$, and $12^{\prime \prime}$, respectively. We use the all-sky atlas images and the WISE Source Catalog to investigate the regions of our INSs. We cross-checked for astrometric shifts between the positions of known 2MASS sources or sources in our previous $H$-band VLT observations and source positions in WISE bands, as well as for noticeable astrometric shifts between WISE sources and the Herschel sources. While the astrometric calibrations of the first three WISE bands generally agree well with NIR astrometry, there are often noticeable shifts in the fourth band which are most easily seen when comparing the last two WISE bands. Astrometric biases in the WISE all-sky atlas images are discussed on the IPAC webpage 14 . While we suspect a shifted W4 astrometry for several of our fields, we do not attempt to improve the $\mathrm{W} 4$ astrometric calibration because the scarcity, faintness and spatial extent of the W4 sources hinder a good, unique astrometric calibration. We ignore the $\mathrm{W} 4$ band in the following.

We searched for potential WISE counterparts of the INSs or neighboring Herschel sources in the remaining bands and discuss our findings in the respective subsections. In Table 4 we provide the WISE field limits measured in Vega magnitudes. The limits correspond to the requirement that the IR sources have signal-to-noise ratios $\mathrm{SNR} \geq 5$ in the respective band. To obtain these limits, we considered all WISE sources in a box area $1^{\circ} \times 1^{\circ}$ around the INS (usually around 20000 sources). Sources with $\mathrm{SNR}=5$ usually have a magnitude uncertainty of $\approx 0.22$ mag.

To convert the Vega magnitudes into flux density units such as Jy, one has to know and account for the source spectrum in the respective WISE band by applying a color correction. Due to the wide WISE bands, these

\footnotetext{
14 wise2.ipac.caltech.edu/docs/release/allsky/expsup/sec2_3g.html
}

Table 4

WISE NS field limits

\begin{tabular}{|c|c|c|c|c|c|c|}
\hline Object & $\begin{array}{c}\mathrm{W1} \\
3.4 \mu \mathrm{m} \\
{[\mathrm{mag}]}\end{array}$ & $\begin{array}{c}\mathrm{W} 2 \\
4.6 \mu \mathrm{m} \\
{[\mathrm{mag}]}\end{array}$ & $\begin{array}{c}\text { W3 } \\
12 \mu \mathrm{m} \\
{[\mathrm{mag}]}\end{array}$ & $\begin{array}{l}\text { W1 } \\
\text { for a } \\
{[\mu \mathrm{Jy}]}\end{array}$ & $\begin{array}{c}\text { W2 } \\
\text { 3B with } \\
{[\mu \mathrm{Jy}]}\end{array}$ & $\begin{array}{c}\mathrm{W} 3 \\
T=200 \mathrm{~K} \\
{[\mu \mathrm{Jy}]}\end{array}$ \\
\hline RXJ0420 & 18.4 & 17.0 & 12.8 & 6 & 20 & 220 \\
\hline $\mathrm{RX}$. & 17.2 & 15.7 & 11.4 & 21 & 67 & 837 \\
\hline RX J0806 & 17.5 & 16.3 & 12.2 & 15 & 38 & 383 \\
\hline RX J1308 & 17.3 & 15.9 & 11.7 & 18 & 56 & 595 \\
\hline J1605 & 17.7 & 16.2 & 12.1 & 12 & 41 & 435 \\
\hline RX J1856 & 17.0 & 15.5 & 11.2 & 24 & 80 & 952 \\
\hline 848 & 15.3 & 14.3 & 11.0 & 110 & 240 & 1145 \\
\hline RX J2143 & 17.3 & 15.8 & 11.6 & 18 & 61 & 690 \\
\hline
\end{tabular}

Note. - Vega magnitude limits in the field of the NSs considering WISE detections with a SNR $>5$. The last three columns show the color-corrected WISE fluxes if a $200 \mathrm{~K}$ blackbody emitter is assumed for the spectral shape.

color corrections can be very different for stars and much cooler blackbody-like sources (asteroids, disks); see, e.g., Cutri et al. (2012), Section IV.4.h; Wright et al. (2010). As an example, we give fluxes for a $T=200 \mathrm{~K}$ blackbody additionally to the Vega magnitudes in Table 4 . but caution that flux limits should in principle be more carefully calculated for any specific (disk) models under investigation.

\section{RESULTS}

There is no significant emission in the WISE, Spitzer, Herschel bands for six of the eight investigated NSs. In Sections 5.1 and 5.2. we present detailed results for the two NSs where significant Herschel red band emission was detected near the location of the NS. Appendix A includes finding charts and measurement details on the six NSs having only upper limits. We summarize our Herschel and color-corrected Spitzer flux (limit) results in Table 5 and the WISE field magnitude limits in Table 4

\section{1. $R X J 0806.4-4123$}

The Spitzer IRAC2 $(4.5 \mu \mathrm{m})$ observation of RX J0806.4-4123 is well aligned with previous NIR observations (e.g, 2MASS point sources), having a $1 \sigma$ astrometric accuracy of $0^{\prime \prime} 2$ (using 196 AAA 2MASS sources). Posselt et al. (2009) investigated VLT ISAAC 


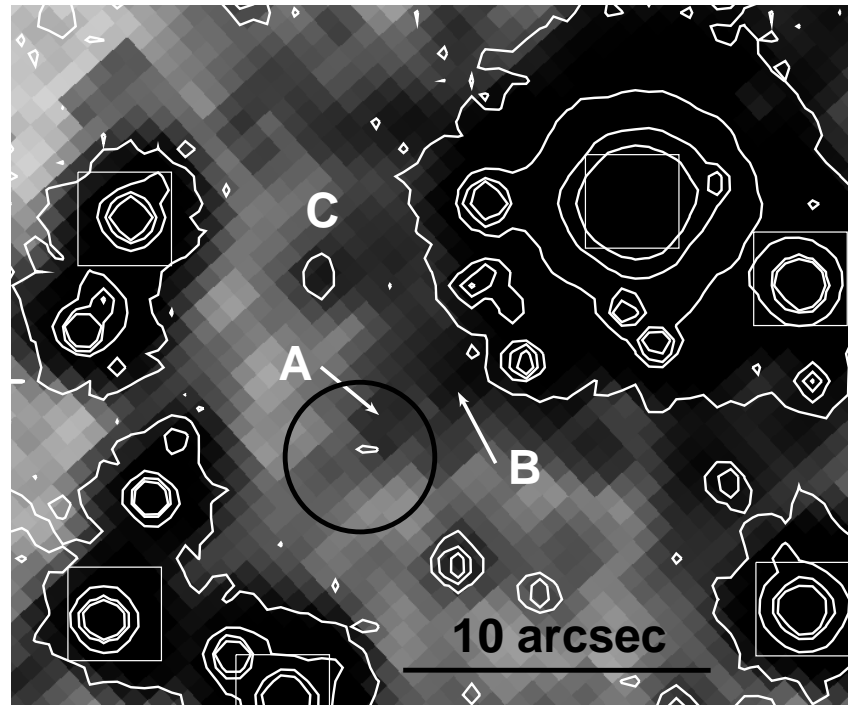

Figure 1. Spitzer IRAC2 $(4.5 \mu \mathrm{m})$ map around RX J0806.44123. The image is $27^{\prime \prime} \times 23^{\prime \prime}$, North is up, East to the left. White contours are from our previous VLT $H$-band observations (Posselt et al. 2009). The white boxes indicate positions of 2MASS point sources, illustrating the good astrometric calibration. The INS position is marked with a black circle with a radius of 2 native IRAC pixels (4 image pixels, $2.4^{\prime \prime}$ ). Note that the astrometric uncertainty for the expected RX J0806.4-4123 position is less than $1^{\prime \prime}$. The color scales of all Figures in this Section have been manually tweaked to emphasize faint fluxes in the surrounding of the NS position. None of these Figures is smoothed.

$H$-band images of this region. Figure 1 shows that the major $H$-band sources correspond well to the Spitzer sources. The Chandra X-ray position of RX J0806.44123 has a positional uncertainty of $00^{\prime \prime} 6$ (90\% confidence, Haberl et al. 2004). Together with the upper limit on the INS proper motion at the time of the Spitzer observation ( $0^{\prime \prime} 88(2 \sigma)$, Motch et al. 2009), the overall positional uncertainty of the expected RX J0806.4-4123 position in IRAC2 is about $0 . \prime 9$ (90\% confidence level) in the worst case.

There appears to be faint emission in the $4.5 \mu \mathrm{m}$ band within this range in the north-west direction of the nominal INS position, see source A in Figure 1. The emission is so faint that we were not able to detect it with the MOPEX PRF-fitting methods. It may be spurious emission; however, there are similarly faint sources which correspond to known NIR sources (e.g., source C in Figure 10. We were not able to produce a reasonable apex-qa residual mosaic in which the brighter sources would be reliably subtracted. As the $H$-band contours show, the IRAC2 emission may contain several IR sources which are not recognized by the PRF-fit. For example, it would be useful to subtract source $\mathrm{B}$, but this source is not even recognized as an individual source in our PRF fits. If we do flux aperture measurements at the INS position (with radius of 2 native IRAC pixels), we obtain nominally an aperturecorrected flux of $F^{4.5 \mu \mathrm{m}}=5.2 \pm 1.9(1 \sigma) \mu \mathrm{Jy}$. Since one can argue about the reliability of such $2.8 \sigma$ emission, we also estimated the aperture-corrected upper flux limit $F_{5 \sigma}^{4.5 \mu \mathrm{m}}=14.7 \mu \mathrm{Jy}$. We note that there is a very faint NIR source at the INS position in the 2004 VLT $H$-band data too, but the NIR emission is below the $3 \sigma$ significance level as well (Posselt et al. 2009).

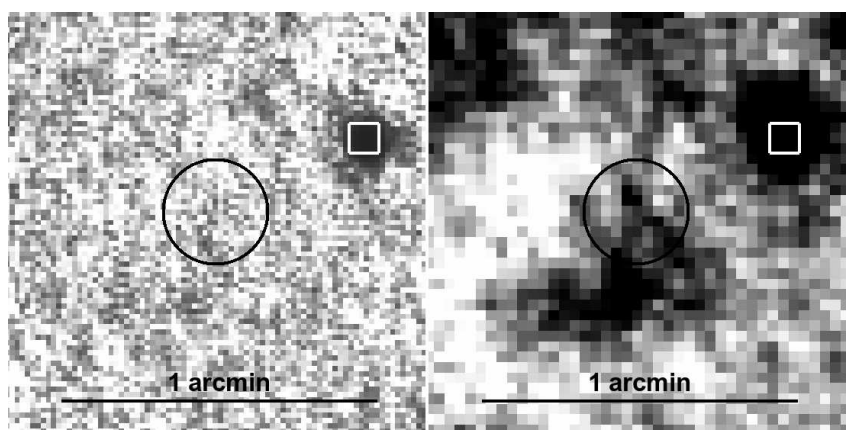

Figure 2. Herschel PACS maps around RX J0806.4-4123. The blue $(60-85 \mu \mathrm{m})$ and red $(130-210 \mu \mathrm{m})$ bands are shown in the left and right panels, respectively. Each map is $1 ! 3 \times 1 ! 3$, North is up, East is to the left. The circle with a radius of $10^{\prime \prime}$ shows the NS position. The white square marks a common source in Herschel PACS, WISE and VLT ISAAC images.

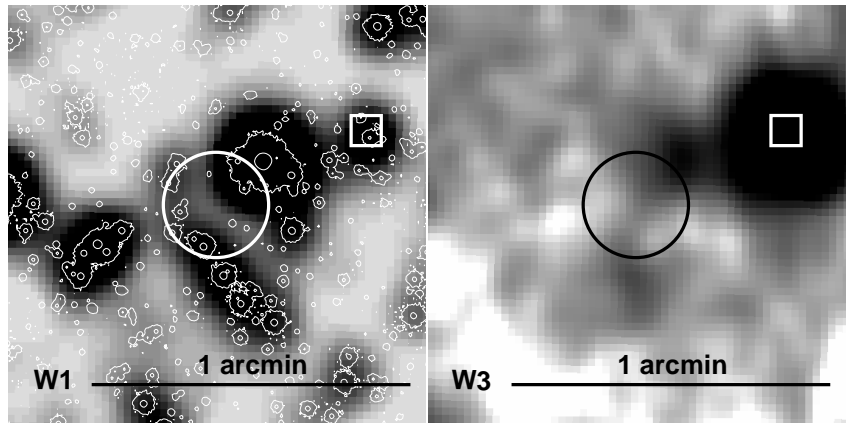

Figure 3. WISE W1 $(3.6 \mu \mathrm{m})$ and W3 $(12 \mu \mathrm{m})$ maps around the position of RX J0806.4-4123. The same field of view is shown as in the Herschel PACS maps of Figure2 2 The white contours in the W1 image are from the VLT ISAAC $H$-band image (Posselt et al. 2009). The white square marks a common source in Herschel PACS, WISE and VLT ISAAC images.

In the Herschel PACS blue band, there is no significant emission around the position of RX J0806.4-4123. Using apertures in different source-free regions and at the target position, we determined the $5 \sigma$ upper flux limit for the INS as $F_{5 \sigma}^{\text {blue }}=4.9 \mathrm{mJy}$. There is, however, emission around the INS position in the Herschel PACS red band, see Figure 2, We use the X-ray position of RX J0806.44123 from Haberl et al. (2004). This position has an uncertainty of 0 "' 6 (90\% confidence). In addition, an uncertainty of 0.83 needs to be considered due to the $2 \sigma$ upper

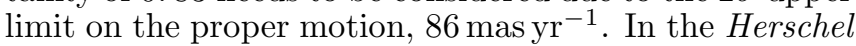
field of view, there is only one unambiguous point source in common with other infrared data, e.g., VLT $H$-band or WISE. The positions of this point source agree well. Comparing the extended emission with the WISE data, we conclude that the nominal Herschel PACS pointing accuracy of $1 \sigma \approx 1_{1}^{\prime \prime} 1$ can be assumed. The PACS red emission around the INS could either consist of several point sources or include extended emission. The peak closest to the INS has a spatial separation of $\approx 2 . .5$ from the X-ray position. We use a $5^{\prime \prime}$ aperture for the flux 
measurement and 10 other apertures on source-free regions to measure the background. Using an aperture correction of 3.4, we estimate a flux of $F^{\text {red }} \approx 10 \pm 5(5 \sigma) \mathrm{mJy}$ at the position of the INS. In Section 6.1 we discuss the likelihood of the Herschel PACS red band emission being not associated with the NS.

We use the previous $H$-band image for cross-checks with the WISE W1-W3 data, see Figure 3 There is no noticeable astrometric shift between these bands. There is no apparent counterpart in the WISE data to the emission in the Herschel PACS red band. Higher spatial resolution is required to investigate the Herschel emission around the INS position.

\section{2. $R X J 2143.0+0654$}

The position of RX J2143.0+0654 is known with an accuracy of 0". 2 from optical observations (Schwope et al. 2009), but its proper motion is unknown. Comparing the Chandra, XMM-Newton, and optical positions from different epochs as shown by Schwope et al. (2009), one can infer that the proper motion must be smaller than 700 mas yr$^{-1}$. Thus, at the time of Herschel and Spitzer observations, the INS could have moved from the optical position by $<33^{\prime \prime} 2$ and $<33^{\prime \prime} 7$, respectively.

There is no emission at the position of RX J2143.0+0654 in the Spitzer IRAC $4.5 \mu \mathrm{m}$ mosaic image, see Figure 4 There are, however, four Spitzer sources within $<3$ '. 7 of the nominal NS position. Since the same sources were detected as faint $H$-band sources in the 2003 VLT observations by Posselt et al. (2009), none of them can be the NS counterpart. The aperture corrected $5 \sigma$ upper flux limit at the nominal NS position is $F_{5 \sigma}^{4.5 \mu \mathrm{m}}=3.0 \mu \mathrm{Jy}$.

The field around RX J2143.0+0654 is densely populated in the infrared and includes several common sources in the Herschel PACS bands, Spitzer IRAC, the WISE W1 \& W2 bands, and in the VLT ISAAC $H$-band observations of Posselt et al. (2009); see Figures 4 to 6. This allows us to confirm that the absolute pointing error of the Herschel observation is not larger than the expected value of 1 .'1.

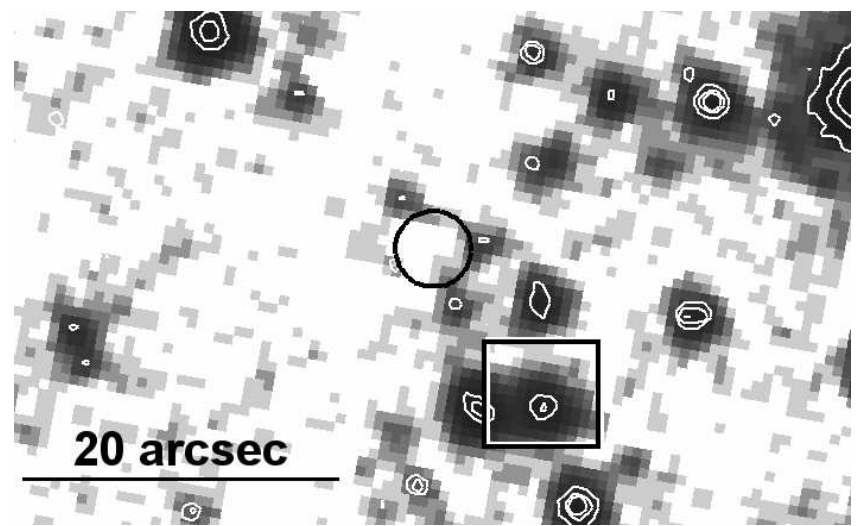

Figure 4. Spitzer IRAC2 $(4.5 \mu \mathrm{m})$ map around the position of RX J2143.0+0654. The white contour highlight sources in the $H$ band (Posselt et al. 2009). The black circle with a radius of 2."4 marks the INS position, the square marks a bright source common in the Herschel PACS, WISE W1 and W2, and $H$-bands.

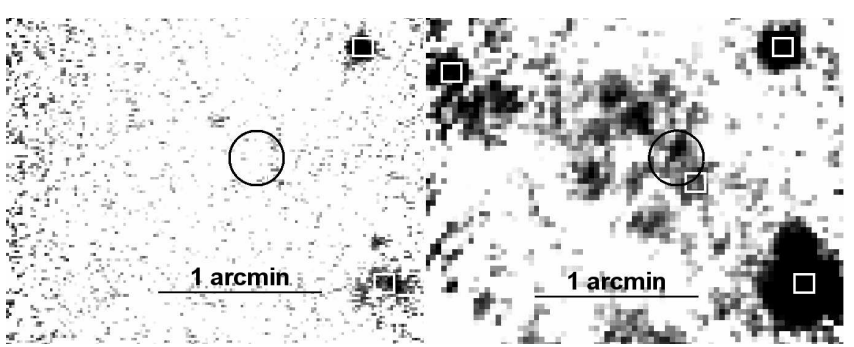

Figure 5. Herschel PACS maps around RX J2143.0+0654. The blue $(60-85 \mu \mathrm{m})$ and red $(130-210 \mu \mathrm{m})$ bands are shown in the left and right panels, respectively. Each map is $2.5 \times 2^{\prime}$, North is up, East is to the left. The circle with a radius of $10^{\prime \prime}$ marks the INS position, the white squares mark bright sources common in the Herschel PACS, WISE W1 and W2, and $H$-bands.

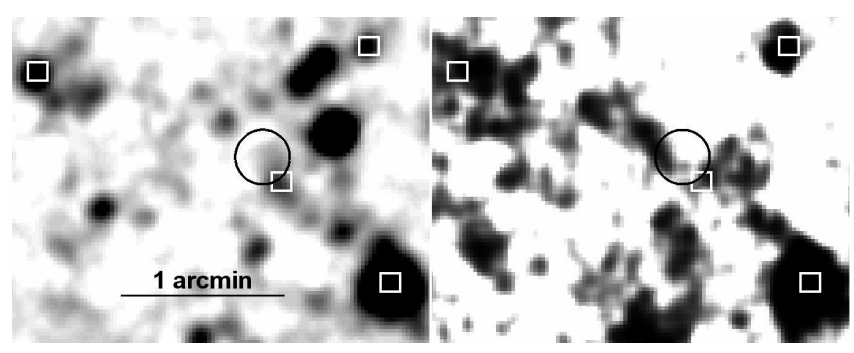

Figure 6. WISE $\mathrm{W} 1(3.6 \mu \mathrm{m})$ and $\mathrm{W} 3(12 \mu \mathrm{m})$ maps around the position of RX J2143.0+0654. The same field of view is shown as in the Herschel PACS maps of Figure 5 The circle with a radius of $10^{\prime \prime}$ marks the INS position, the white squares mark bright sources common in the Herschel PACS, WISE W1 and W2, and $H$-bands.

There is emission in the red PACS band at the position of the INS, consisting of a blend of at least one southern and one brighter northern source (see Figure 5, the southern source is marked with a box and was also detected by WISE). In the blue PACS band, there is very faint emission northwest of the INS. The peak position of the $160 \mu \mathrm{m}$ emission is 3.7 north of the optical INS position, about 2".6 from the closest N(IR) source. Considering the unknown NS proper motion, we cannot ruled out the possibility that this source is the counterpart of the INS. However, it appears unlikely that RXJ2143.0+0654 has the implied large proper motion $\left(>0^{\prime \prime} 6 \mathrm{yr}^{-1}\right)$. While the INS distance is unknown, it is expected to be larger than $300 \mathrm{pc}$ (Posselt et al. 2007). For comparison, the closest of the XTINSs, RX J1856.5-3754 ( $D=140 \mathrm{pc})$ has also the largest proper motion (330 $\mathrm{mas} \mathrm{yr}^{-1}$ ) of those four of the XTINSs for which proper motion measurements or limits were reported.

A part of the red band emission at the INS position is likely to come from the bright infrared southwestern source which is seen in several bands. Given the proximity of the sources, their faintness and the FWHM in the red band of $10^{\prime \prime} .5 \times 12^{\prime \prime} .02$, it is not possible to remove the sources with the necessary accuracy to search for residual $160 \mu \mathrm{m}$ emission at the optical position of the INS. We used an aperture with radius of $5^{\prime \prime}$ at the position of INS to measure the flux at this position. The aperture-corrected flux is $F^{\text {red }}=7.8 \pm 4.8(5 \sigma) \mathrm{mJy}$. Due to the surrounding sources, this value must be regarded with caution and it can be an overestimate of any actual 
Table 5

Flux measurements and limits

\begin{tabular}{lccc|rr}
\hline Object & $\begin{array}{c}\mathrm{F}^{4.5 \mu m} \\
{[\mu \mathrm{Jy}]}\end{array}$ & $\begin{array}{c}\mathrm{F}^{\text {blue }} \\
{[\mathrm{mJy}]}\end{array}$ & $\begin{array}{c}\mathrm{F}^{\text {red }} \\
{[\mathrm{mJy}]}\end{array}$ & $\begin{array}{r}\mathrm{M}_{\text {dust }}^{20 \mathrm{~K}} \\
{\left[\mathrm{M}_{\oplus}\right]}\end{array}$ & $\begin{array}{r}\mathrm{M}_{\text {dust }}^{\text {100K }} \\
{\left[\mathrm{M}_{\oplus}\right]}\end{array}$ \\
\hline RXJ0420 & $<2.1$ & $<4.5$ & $<7.0$ & $<1.0$ & $<0.02$ \\
RX J0720 $^{\mathrm{a}}$ & $<3.5$ & $<5.2$ & $<9.4$ & $<1.4$ & $<0.02$ \\
RX J0806 $_{\text {RX J1308 }}<11.4^{\text {PD }, \mathrm{CN}}$ & $<4.9$ & $10 \pm 5 \mathrm{CN}$ & $0.72 \pm 0.36$ & $0.012 \pm 0.006$ \\
RX J1605 & $<1.2$ & $<1.7$ & $<5.2$ & $<1.5$ & $<0.02$ \\
RX J1856 & $<2.7$ & $<6.1$ & $<12.2$ & $<2.2$ & $<0.04$ \\
PSR J1848 & $<8.9^{\mathrm{CN}}$ & $<7.7$ & $<7.8$ & $<0.2$ & $<0.004$ \\
RX J2143 & $<$ & $<3.6$ & $<5.9$ & $<6.2$ & $<0.1$ \\
\hline
\end{tabular}

Note. - The results of the following bands are listed: Spitzer IRAC2 $(4.5 \mu \mathrm{m})$, Herschel PACS blue band (60-85 $\mu \mathrm{m})$, and red band $(130-210 \mu \mathrm{m})$. For Spitzer data, the color corrections for a $T=200 \mathrm{~K}$ blackbody are applied, but note the general remarks regarding color corrections in Section 4 The superscript ${ }^{\mathrm{PD}}$ indicates a potential (weak) detection, ${ }^{C N}$ indicates confusing neighbor sources. Dust masses are calculated using Equation (1) for dust temperatures of $20 \mathrm{~K}$ and $100 \mathrm{~K}$, the Herschel PACS red band measurements, and the distances as listed by Kaplan \& van Kerkwijk (2009). All limits and errors are $5 \sigma$ values.

${ }^{a}$ RX J0720 also has a Spitzer IRAC1 $(3.6 \mu \mathrm{m})$ limit, $F^{3.6 \mu m}<3.2 \mu \mathrm{Jy}$ (color corrected for $\left.T=200 K\right)$.

flux from the INS position. In Section 6.1 we discuss the likelihood of the Herschel PACS red band emission being not associated with the NS.

In the blue band there is no prominent source emission at the INS position. Using apertures in different sourcefree regions and at the target position, we determined the $5 \sigma$ upper limit for the RX J2143.0+0654 flux as $F_{5 \sigma}^{\text {blue }}=5.0 \mathrm{mJy}$.

We used the VLT ISAAC $H$-band image by Posselt et al. (2009), Spitzer, and 2MASS point sources for cross-checks with the WISE W1-W3 data, see Figures 4 and 6. There is no noticeable astrometric shift between these bands. The W1 emission west of the INS position is probably related to the southwestern sources seen with Spitzer, and no enhanced emission is detected at the INS position. In W3, there is no emission peak at the INS position, although there is an eastern source at $\approx 8^{\prime \prime}$ and a faint southern source at $\approx 5^{\prime \prime}$ from the nominal NS position. Since the astrometry of sources in these WISE images agree well with those of $\mathrm{N}(\mathrm{IR})$ sources, we conclude that RX J2143.0+0654 is undetected in the WISE W1-W3 data.

\section{DISCUSSION}

6.1. The likelihood for the Herschel emission to be associated with field sources

In Sections 5.1] and 5.2, we reported on Herschel red band emission at or near the positions of RX J0806.44123 and RX J2143.0+0654. How likely is the emission in the Herschel PACS red band associated with a source different from the INS ? Confusion from faint Galactic stars is negligible for the PACS red band 15 . The main two components of sky background confusion noise in the long-wavelength bands of Herschel are extragalactic sources and interstellar cirri. Sibthorpe et al. (2013) showed that, for a given Herschel PACS flux level, results from typical extragalactic fields can be used to estimate the background source numbers in typical debris disk surveys. They used the results from the PACS Extragalactic Probe (Berta et al. 2011; Lutz et al. 2011) to estimate the expected background source number

${ }^{15}$ herschel.esac.esa.int/Docs/HCNE/pdf/HCNE_ScienceDoc.pdf density for given flux and flux uncertainty levels. Following the approach by Sibthorpe et al. (2013), we estimate the chance probability of detecting an $F_{\text {red }} \approx 10 \mathrm{mJy}$ extragalactic source in a circle with radius of $r=5^{\prime \prime}$ as $0.5 \%$. For RX J2143.0+0654, we estimate the respective chance probability of detecting an $F_{\text {red }}=7.8 \mathrm{mJy}$ extragalactic source in a circle with radius of $r=5^{\prime \prime}$ as $0.7 \%$. Given these chance probabilities, the binomial probability that we detect background sources in two of overall eight XTINS fields is at maximum $1 \%$, for $7.8 \mathrm{mJy}$ sources. But the probability to detect one background galaxy at one of the eight XTINS positions is already $5 \%$.

Gáspár \& Rieke (2014) argued that Sibthorpe et al. (2013) underestimate the confusion noise probabilities, mainly because galaxies fainter than a considered flux limit may also contribute to the overall confusion noise. We used the Monte Carlo Code by Gáspár \& Rieke (2014) to estimate the probabilities for $N_{\text {excess }}$ unrelated 'excess sources' in the photometry apertures of our eight NSs. We realized $10^{6}$ datasets of eight sources with our detection requirement $\gtrsim 5 \sigma$ (Table 5. i.e., $5 \mathrm{mJy}$ for RX J0806.4-4123) in a $r=5^{\prime \prime}$ photometry aperture. In a nutshell, the code by Gáspár \& Rieke (2014) simulates background sources in a single large field (0.5 square degrees), then properties for 8 random positions from this field are estimated $10^{6}$ times. The latter step includes the consideration of noise from interstellar cirri by using a probability distribution with standard deviation $\sigma_{\text {cirrus }}$ which is calculated from the observed far-infrared ISM flux background. For details on the code and the assessment of its statistical performance, we refer to Gáspár \& Rieke (2014). For our simulations, we used a cirrus noise value of $\sigma_{\text {cirrus }}=0.84 \mathrm{mJy}$, which corresponds to the median value of the ISM background flux of $11.74 \mathrm{MJysr}^{-1}$, as estimated by using Hspot 16 for the observing dates and positions of our eight NSs. The resulting probability plot is shown in Figure 7 . The probability to detect no, one or two excess source(s) among our eight NSs is $86 \%, 13 \%$ and $1 \%$, respectively. Thus, it seems unlikely that both our detections are excess sources.

16 The Herschel tools are available at: herschel.esac.esa.int/Tools.shtml 


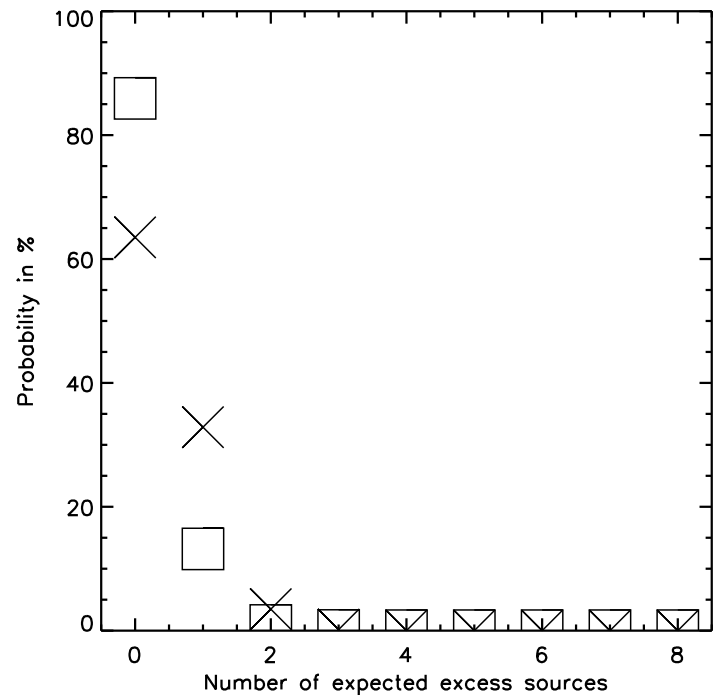

Figure 7. The probability of detecting $N_{\text {excess }}$ excess sources in our investigated NS sample. We use the Monte Carlo code by Gáspár \& Rieke (2014), simulating $10^{6}$ datasets of eight sources with our detection requirement $\gtrsim 5 \sigma$ in a $r=5^{\prime \prime}$ photometry aperture. Boxes indicate the values for using the median interstellar cirrus noise level of our eight NS fields. Crosses indicate the results of the simulation which takes into account the exceptionally high ISM background flux for RX J0806.4-4123.

Kaplan et al. (2011) investigated RX J0806.4-4123 with the Hubble Space Telescope (HST) and found no source other than the NS within $2^{\prime \prime}$ of the INS position using the Advanced Camera for Surveys, Wide Field Channel/filter F475W down to a ST magnitude of $27.92 \pm 0.22$. Considering the Herschel pointing accuracy (1".1, Section 5.1), its large FWHM $\left(\approx 11^{\prime \prime}\right.$ for the red band), there are, however, several HST sources which currently cannot be excluded as potential Herschel background galaxy counterparts.

There is also another alternative explanation for the Herschel PACS red band source: interstellar cirrus. In fact, the ISM background flux in the direction of RX J0806.4-4123 is estimated by Hspot to be about a factor 10 higher than the median value of the remaining seven NS fields. Illustrating the higher ISM background, the recent 3D maps of the local ISM distribution by Lallement et al. (2014) show a local ISM cloud clump in the direction of RX J0806.4-4123. A typical interstellar cirrus has been found to have temperatures of about $\sim 20 \mathrm{~K}$ with a range of about $4 \mathrm{~K}$ around this value (Veneziani et al. 2013, see also Gáspár \& Rieke 2014) a temperature range very reminiscent of the one inferred for our Herschel detection around RX J0806.4-4123 (see section 6.2). If we consider explicitly the higher ISM background flux for this one source in the Monte Carlo simulation by Gáspár \& Rieke (2014), we obtain $63 \%, 33 \%$ and $3 \%$ as the probabilities for detecting no, one, or two excess source(s) among our eight NSs, respectively. The probability of one excess source among the eight NSs has nearly tripled, but the probability for no excess source in the sample is still a factor 2 higher.

In the case of RX J2143.0+0654, the HST observations are not constraining with respect to excluding obviously present galaxies as counterparts to the faint Herschel emission either. Regarding interstellar cirri, the ISM background flux in the direction of RX J2143.0+0654 is nearly the same as the median value for the seven NS fields (excluding RX J0806.4-4123). Thus, the probability for an excess source due to an ISM cirrus is less than in the case of RX J0806.4-4123.

\subsection{Multi-wavelength constraints on the dust emission}

In the following, we assume that the detected Herschel emission originates from dust associated with the XTINS. We summarize the Herschel and Spitzer results of the previous subsections in Table 5. Assuming the emitting dust to be optically thin at submillimeter wavelengths, we can use the Herschel $160 \mu \mathrm{m}$ measurements to calculate the dust mass assuming a single temperature for all dust grains:

$$
M_{d}=\frac{F_{\nu} D^{2}}{B_{\nu}\left(T_{d}\right) \kappa_{\nu}^{d}}
$$

where $\nu=1.9 \times 10^{12} \mathrm{~Hz}$ is the frequency, $F_{\nu}$ is the measured flux density (limit), $D$ is the distance, $B_{\nu}\left(T_{d}\right)$ is the Planck function at a dust temperature $T_{d}$, and $\kappa_{\nu}^{d}$ is the dust mass absorption coefficient. We use $\kappa_{160 \mu \mathrm{m}}^{d}=13 \mathrm{~cm}^{2} \mathrm{~g}^{-1}$ for the PACS red band, which we calculated from the optical constants by Dorschner et al. (1995) assuming dust grains consisting of amorphous magnesium silicate with a grain density $\rho_{g}=2.7 \mathrm{~g} \mathrm{~cm}^{-3}$. The dust composition and, therefore, the dust mass absorption coefficient is in general highly uncertain (by a factor 3 to 5, e.g., Posselt et al. 2010). Additionally, the dust likely has a temperature distribution, not a single temperature. The composition and dimensions of a fallback disk around $\mathrm{a} \sim 1 \mathrm{Myr}$ old NS are unknown, too. Thus, the derived dust masses in Table 5 and Figure 9 are only crude estimates.

In Figures 8 and 9, we show the ratios of the $\mathrm{IR} /$ submillimeter fluxes (limits) to the X-ray fluxes of the XTINSs. The obtained flux ratios (limits) are usually in the range $10^{-4}$ to $10^{-3}$ for the $H$-band and the Spitzer $4.5 \mu \mathrm{m}$ data, but 0.01 to 0.1 for the Herschel data. For comparison, we refer to similar plots for three CCOs by Wang et al. (2007a) (for optical and IR fluxes only). At the Spitzer wavelengths, flux ratio limits of $10^{-2}$ to $10^{-4}$ were reached for the CCOs, but no disks were detected. Wang et al. (2007a) also showed a comparison plot for the only magnetar where a disk is thought to be detected - 4U 0142+61 (Wang et al. 2006). For this magnetar, the flux ratio at Spitzer IRAC wavelengths is $\approx 6 \times 10^{-5}$. This comparison might indicate that our limits are simply not deep enough. On the other hand, the single object 4U $0142+61$ might not be defining for all potential NS disks. Our Herschel detections of emission around RX J0806.4-4123 and RXJ2143.0+0654 could indicate predominantly cold dust around these (older) NSs. Spitzer $4.5 \mu \mathrm{m}$ observations are not sensitive enough to detect such cold dust.

Dust emission at long wavelengths can be described in the Rayleigh limit if $\lambda>\max \left(2 \pi a, \lambda_{\mathrm{Res}}\right)$, where $a$ is the (spherical) grain size radius, and $\lambda_{\text {Res }}$ is the largest resonance wavelength of the complex refractive index as 


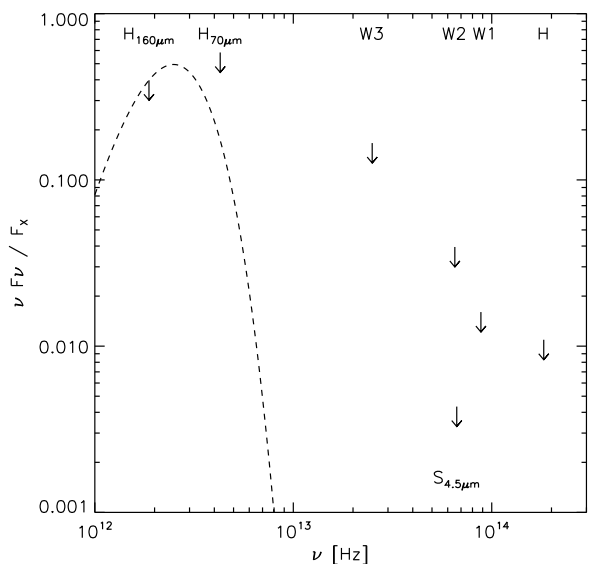

RX J1605.3+3249

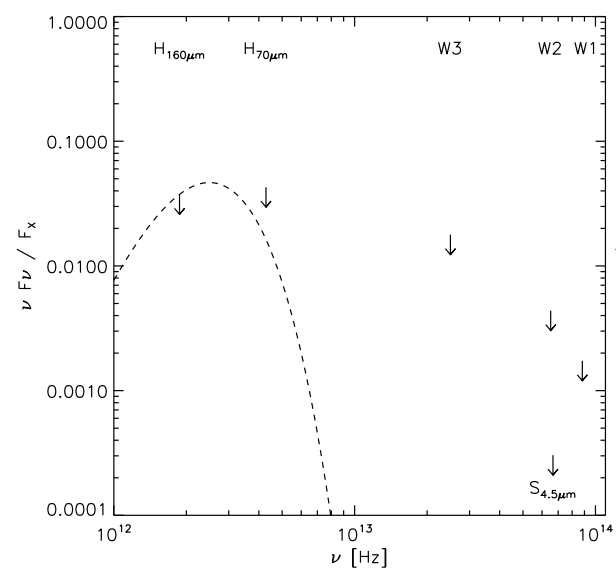

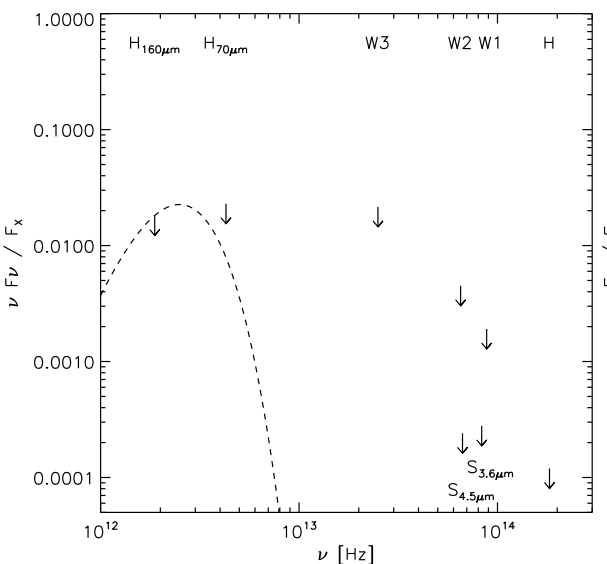

RX J1856.5-3754

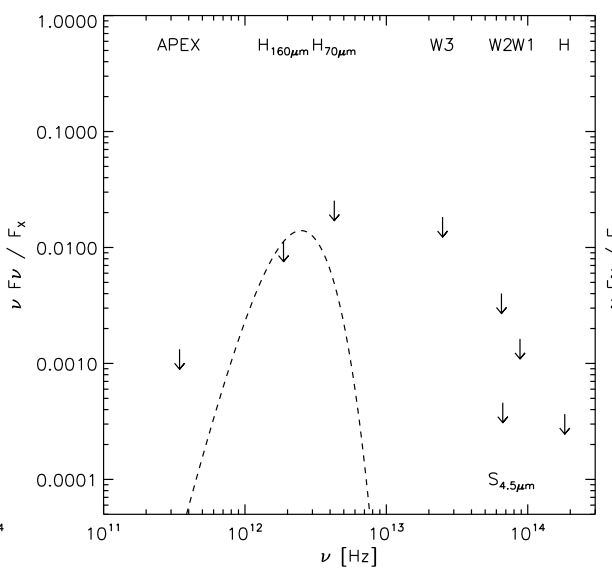

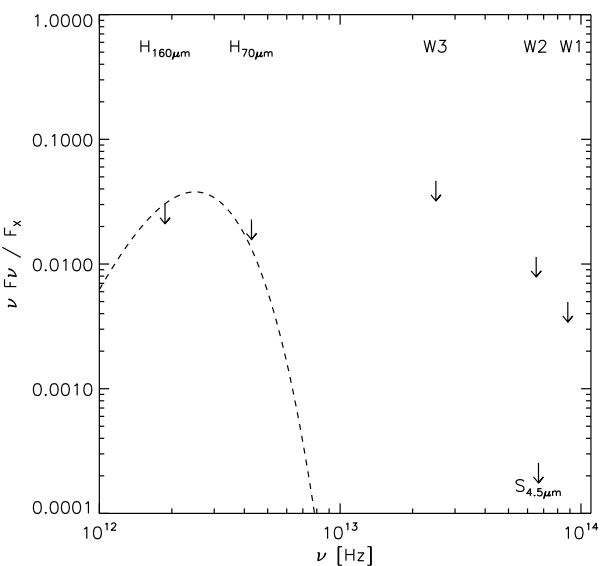

RX J2143.0+0654

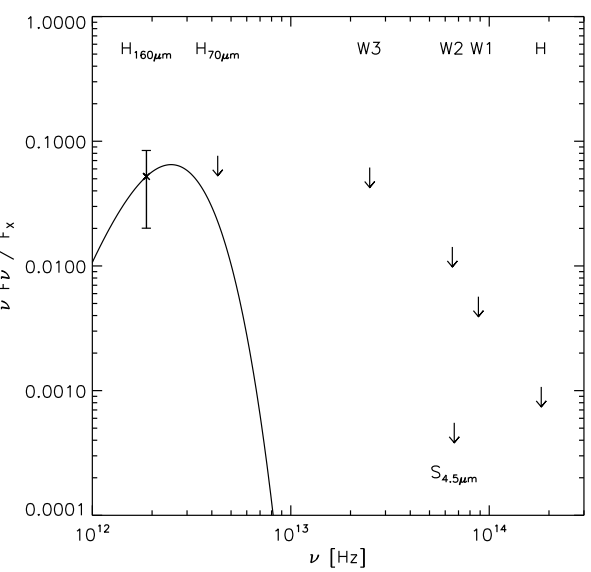

Figure 8. Measured values and upper limits for the IR/submillimeter to X-ray flux ratios for six XTINSs. The X-ray fluxes are for the energy range $0.1-2.4 \mathrm{keV}$ and are taken from Haberl (2013). Note that the XTINSs show only very soft thermal X-ray emission. The infrared/submillimeter limits/values are taken from Tables 4 and 5 and the measurements by Posselt et al. (2010, 2009). For Spitzer and WISE data, exemplary color corrections for a $T=200 \mathrm{~K}$ blackbody are applied, but note the general remarks regarding color corrections in Section 4 No extinction correction has been applied since the XTINSs have very small absorbing hydrogen column densities $\left(N_{H} \approx 10^{20} \mathrm{~cm}^{-2}\right)$, corresponding to negligible extinction corrections in the Infrared $\left(A_{H} \approx 0.01, A_{3.6 \mu \mathrm{m}} \approx 0.004\right.$; following the extinction relations by Vuong et al. 2003 and Indebetouw et al. 2005). The dashed lines show upper limits on the emission of dust with a temperature $T_{d}=20 \mathrm{~K}$ according to Equation (2). The solid line in the case of RX J2143.0+0654 indicates the respective constraints from the measured Herschel aperture flux.

tabulated in the DOCCDI7. If this condition is fulfilled, the flux density $F_{\nu} \propto \nu^{2}$ (see Appendix [B]), and assuming optically thin conditions for the grain as well as the overall medium (e.g., dusty disk or cloud), we can write:

$$
F_{\nu}=\frac{B_{\nu}\left(T_{d}\right)}{B_{\nu_{0}}\left(T_{d}\right)}\left(\frac{\nu}{\nu_{0}}\right)^{2} F_{\nu_{0}}
$$

where $\nu_{0} \ll 10^{13} \mathrm{~Hz}$ is the reference frequency. Here, we choose the Herschel red band $\left(\nu_{0}=1874 \mathrm{GHz}\right)$ as reference band and show the constraints on the expected dust fluxes at long wavelengths in Figures 8 and 9 . In the former we use an exemplary temperature $T_{d}=20 \mathrm{~K}$, in the latter we illustrate the effect of choosing different temperatures.

In the wavelength regime of W1-W3, Spitzer IRAC and the $H$-band, the dust absorption and emission properties

17 Database of Optical Constants for Cosmic Dust www.astro.uni-jena.de/Laboratory/OCDB/index.html are highly non-monotonic functions of $\lambda$ (see, e.g, the laboratory absorption coefficient measurements of dust grains by Dorschner et al. 1995), and the dust medium is in general not optically thin for (its own) emission at such wavelengths. Realistic flux models would require the application of the Mie theory for the radiation transport calculation for a distribution of dust grains. Given that we deal only with flux limits at these wavelengths, such modeling is beyond the scope of this paper. To estimate at least the maximal upper limits on the luminosity ratios, we assume blackbody emission. For blackbody emission, the dust luminosity limits, $L_{\text {dust }}\left(T_{d}\right)$, are related to the measured flux density limits, $F_{\nu}$,

$$
F_{\nu}=\frac{B_{\nu}\left(T_{d}\right) L_{\mathrm{dust}}}{4 D^{2} \sigma_{S B} T_{d}^{4}},
$$

where $\sigma_{S B}$ is the Stefan-Boltzmann constant. The right plot in Figure 9 shows the blackbody luminosity constraints for RX J0806.4-4123. 

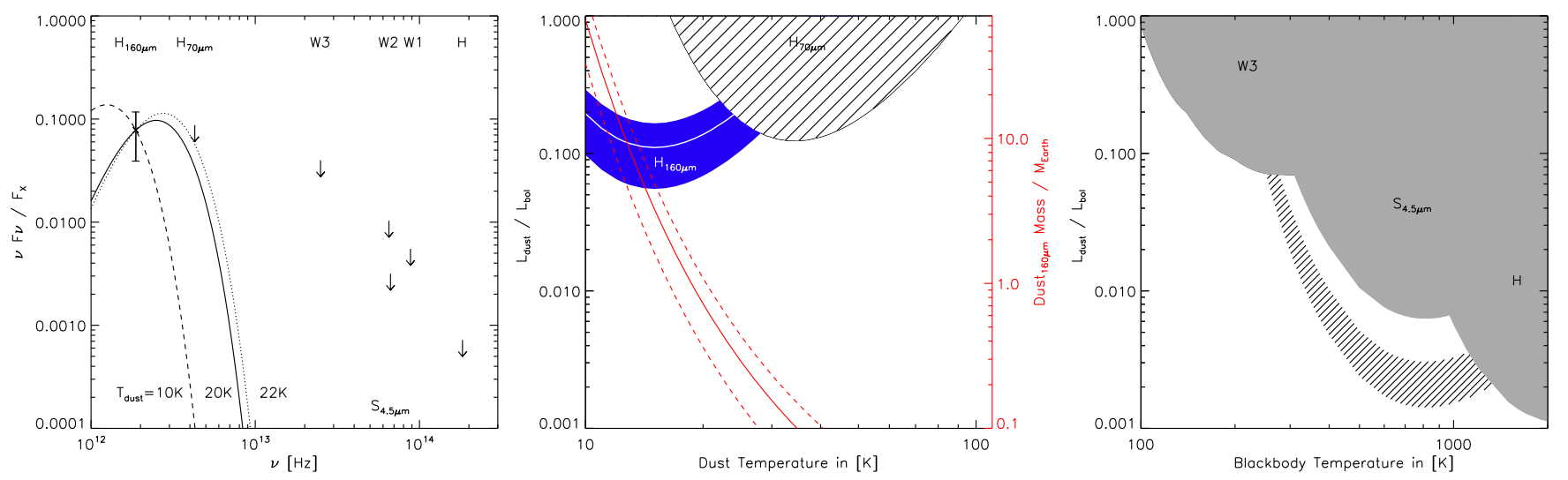

Figure 9. Measured values and upper limits for the IR/submillimeter to X-ray flux ratios for RX J0806.4-4123 (left panel) and constraints on the luminosity and temperature of dust around it (middle and right panel). The flux ratios are from this paper and the literature as described for Figure 8 The luminosity ratios are derived with respect to the bolometric luminosity (which is basically the X-ray luminosity), the mass is calculated using a distance of $250 \mathrm{pc}$ which is based on the X-ray absorption (Kaplan \& van Kerkwiik 2009; Posselt et al. 2007). The middle panel shows the luminosity constraints for dust emission according to Equation (4). The blue area marks the luminosity ratio range implied by the Herschel $160 \mu \mathrm{m}$ measurement (white line) and its $5 \sigma$ uncertainty, the dashed area indicates the excluded dust luminosities according to our Herschel blue band observations. The red solid and dashed lines show the inferred mass constraints from the Herschel $160 \mu \mathrm{m}$ measurement. The right panel shows the upper limit on the luminosity ratios as implied for blackbody emitters under optically thin conditions. The shaded area indicates the excluded region for blackbody emitters. Similarly, the hatched area shows the allowed range for the marginal 1.9 $\sigma$ Spitzer $4.5 \mu \mathrm{m}$ detection, see Section 5.1 Realistic dust disk models require the application of the Mie theory and would result in lower limits in the right panel (see text).

For $h \nu_{0} \gg 2.8 k T$, optically thin dust conditions, and uniform properties of the dust grains as well as an uniform temperature, we derive for the total luminosity of the dust:

$$
\begin{array}{r}
L_{\text {dust }}\left(T_{d}\right)=\frac{160}{21} \pi^{2}\left(\frac{k T_{d}}{h \nu_{0}}\right)^{2} \kappa_{\nu_{0}}^{d} M_{d} \sigma_{S B} T_{d}^{4} \\
=F_{\nu} D^{2} \frac{160}{21} \pi^{2}\left(\frac{k}{h \nu}\right)^{2} \frac{\sigma_{S B}}{B_{\nu}\left(T_{d}\right)} T_{d}^{6},
\end{array}
$$

where $k$ is the Boltzmann constant, and $h$ is the Planck constant. The middle plot of Figure 9 shows the constraints on $L_{\text {dust }}\left(T_{d}\right) / L_{X}^{N S}$ from the Herschel red band value and blue band limit. We see that only low dust temperatures $\left(T_{d}<25 \mathrm{~K}\right)$ are allowed considering the detection and non-detection in the red and blue band, respectively.

\subsection{Constraints on a potential dusty disk or torus}

In the previous Section we discussed the constraints on the dust associated with the XTINSs only in general. Here, we discuss constraints on different properties of potential disks assuming that the detected Herschel fluxes are indeed due to emission from dust around the NSs. Bryden et al. (2006) noted that only the brightest debris disks detected with Spitzer have $L_{\text {dust }} / L_{\text {bol.* } *} \geq 10^{-4}$. The recent Herschel DUNES survey by Eiroa et al. (2013) reported $L_{\mathrm{dust}} / L_{\mathrm{bol}, *}$ ranging from $10^{-6}$ to $3 \times 10^{-4}$ for Herschel debris disk candidates around solar-type stars. If the Herschel $160 \mu \mathrm{m}$ detections for two 2 XTINSs and the marginal Spitzer $4.5 \mu \mathrm{m}$ and $H$-band detections of emission around RX J0806.4-4123 are confirmed to come from disks, the luminosity ratios would be two to three orders of magnitudes higher than the values seen for non-degenerate stars. The implied large absorption cross section of the disk appears questionable. In contrast to the luminosities of main sequence stars, most of the XTINS's luminosity is emitted in soft X-rays $(<1 \mathrm{keV})$. In contrast to other wavelengths, soft X-ray photons are very efficiently absorbed and re-emitted by all dust grains $(\approx 100 \%$ of each incident soft X-ray photon; e.g., Dwek \& Smith 1996). The absorption cross section of a putative disk cannot, however, be larger than the geometric cross section of that disk. Hence, even if one accounts for an exceptionally high dust heating efficiency, geometric arguments exclude a thin flared disk from gobbling up $20 \%$ of the stellar luminosity. The implied geometric shape for an assembly of dust grains surrounding the NS would rather be a dusty torus or an envelope.

We use the Rayleigh approximation to estimate the possible location of the dust around the NS. As outlined in the Appendix B the temperature distribution, $T_{\mathrm{g}}(r)$, of amorphous magnesium silicate dust grains with sizes $a$ can be calculated as:

$$
T_{\mathrm{g}}(r)=39\left(\frac{L_{\mathrm{bol}, 30}}{a_{\mu \mathrm{m}}}\right)^{1 / 6}\left(\frac{1}{r_{14}}\right)^{1 / 3} \mathrm{~K}
$$

where the NS luminosity, $L_{\mathrm{bol}, 30}$, is normalized to $10^{30} \mathrm{erg} \mathrm{s}^{-1}$, and the distance, $r_{14}$, between the NS and the grain is normalized to $10^{14} \mathrm{~cm}$. Note that this equation is only valid for $T<\min \left(96 \mathrm{~K}, 2300 a_{\mu \mathrm{m}}^{-1} \mathrm{~K}\right)$.

As shown in Section 6.2 and Figure 9, the inferred temperature for potential circumstellar dust around, e.g., RX J0806.4-4123 is in the range of 10 to $22 \mathrm{~K}$. A dust grain with such temperatures would be expected at radii of $r_{d}=2.3 \times 10^{16} a_{\mu \mathrm{m}}^{-1 / 2} \mathrm{~cm}$ to $2.2 \times 10^{15} a_{\mu \mathrm{m}}^{-1 / 2} \mathrm{~cm}$, corresponding to $1600 a_{\mu \mathrm{m}}^{-1 / 2}$ A.U. and $150 a_{\mu \mathrm{m}}^{-1 / 2}$ A.U., respectively. At a distance of $\approx 250 \mathrm{pc}$, this translates into angular sizes of about $6^{\prime \prime}$ and $1^{\prime \prime}$, i.e., the source would be still unresolved in the Herschel red band which 


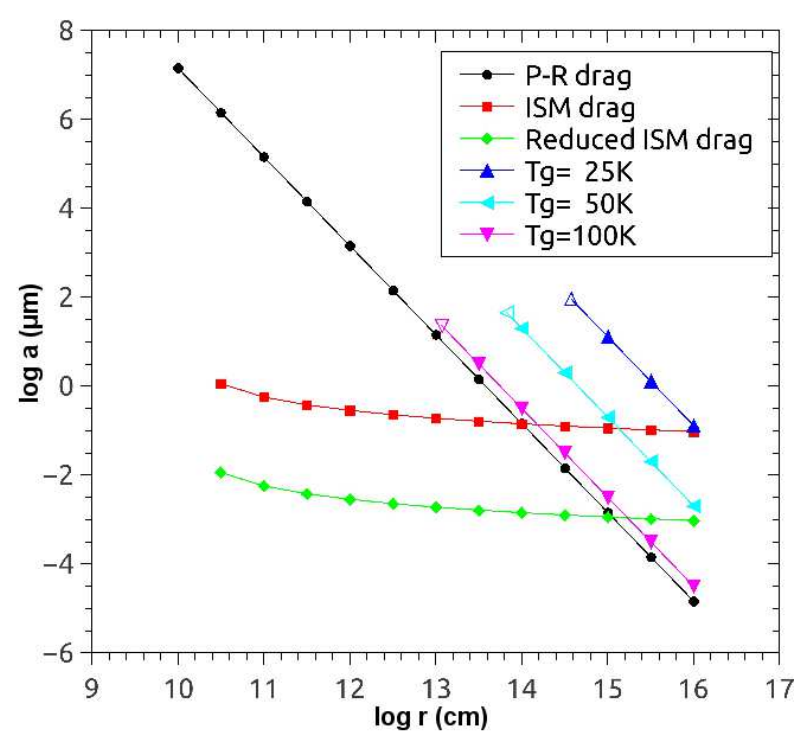

Figure 10. Distribution of spherical grains with radius $a$ in $\mu \mathrm{m}$ over the distance from the NS, $r$ in $\mathrm{cm}$, for different temperatures and dust removal effects. The blue, cyan, and magenta (all with triangles) lines indicate the temperature a dust grain would have if Eq. 5] is applied. The open triangle symbols indicate the region left of which the conditions for Eq. 5 are not met. The black line indicates removal of grains due to the Poynting-Robertson drag. Grains below the black line are expected to be removed at the assumed disk age of 0.5 Myr. Similarly, the green and red lines indicate the effect of the ISM drag the dust grains experience when they move together with the NS disk through the ISM (e.g., Phillips \& Chandler 1994), please see also text.

is consistent with the observations. Thus, we cannot rule out such temperatures based on the expected emission extension for RX J0806.4-4123. For RX J2143.0+0654, the inferred radii would be factor 2.8 larger due to its higher X-ray luminosity, although the angular scales are probably similar because of the likely larger distance of this XTINS. The implied orbital radii of the $22 \mathrm{~K}$ dust grains with sizes $a \lesssim 1 \mu \mathrm{m}$, are larger than the radii commonly considered for (young and gas-rich) fallback disks, $R_{\mathrm{FD}} \approx 10^{10}-10^{14} \mathrm{~cm}$ (e.g., Perna et al. 2014). After removal of the gas in the possible disks around the relatively old INSs, however, the dust is difficult to remove by ISM drag (see also below), and a pure dust or debris disk could, in principle, expand to larger radii. Looking at our own solar system, there are the following reminiscent large structures: the Kuiper belt at $30-60$ A.U. with lateral coverage of up to $40^{\circ}$ (e.g., Brown 2001) and the hypothesized near-spherical Oort cloud with radii from $10^{4}-10^{5}$ A.U. (e.g., Dones et al. 2004). The formation of neither the Kuiper belt nor the Oort cloud are fully understood, but in general it is believed that gravitational interactions of belt/cloud objects with the newly-formed giant planets were of major importance (Morbidelli et al. 2008; Dones et al. 2004). Survival or formation of giant planets around a NS are unlikely, and a mechanism for the extension of an initial compact fallback disk would be needed if the emitting dust grains are smaller than $10 \mu \mathrm{m}$. Constraints on the emitting grain size could be obtained by additional investigations at submillimeter wavelengths.

The smallest grains are the hottest and contribute most to the observed dust luminosity. If, in the case of cicumstellar dust, already $\approx 20 \%$ of the NS luminosity is re-emitted by cold dust, but the upper limit at Spitzer wavelength is less than $1 \%$ (because of blackbody assumption; see Figure 9), it follows that there are no significant amounts of small hot dust grains around RX J0806.4-4123. An explanation for this finding can be the effect of the Poynting-Robertson (P-R) drag (e.g., Phillips \& Chandler 1994). In Figure 10, we plot lines corresponding to the $\mathrm{P}-\mathrm{R}$ drag and different dust temperatures (for the latter we use Eq. 5). The black line corresponds to the P-R drag limit for a putative disk around a NS with radius of $R_{\mathrm{NS}}=10 \mathrm{~km}$ and a disk age of $0.5 \mathrm{Myr}$. Dust grains below that line are removed for the respective radius in a putative disk. We see that small grains close to NS are effectively eliminated. Dust grains with size $\sim 1 \mu \mathrm{m}$ can exist only at radii $r>3 \times 10^{13} \mathrm{~cm}$. In Figure 10, we also plot the effects of the ISM drag experienced by the dust grains when they move together with the NS disk/belt through the ISM (e.g., Phillips \& Chandler 1994). The ISM drag depends on the inclination of the putative disk/belt with respect to the direction of proper motion. We use different inclination angles. If the putative disk moves edge-on in the direction of the proper motion, the inclination angle is $0^{\circ}$. This assumption allows us to obtain an upper limit for the smallest grain size, represented by the red line in Figure 10. An inclination angle of $89^{\circ}$ corresponds to the putative disk moving nearly face-on in the direction of the proper motion. This scenario is more likely because many NSs have a spin-velocity alignment and angles $<10^{\circ}$ are most probable. In Figure 10, this scenario is represented by the green line. We assumed a disk age of $0.5 \mathrm{Myr}$, a NS velocity of $100 \mathrm{kms}^{-1}$, a number density of ISM gas particles $1 \mathrm{~cm}^{-3}$, and a dust grain density of $3 \mathrm{~g} \mathrm{~cm}^{-3}$ for our calculations. The ISM drag is the dominating force on the dust grains at large distances. Similarly to the P-R line, grains below the ISM drag lines in Figure 10 would be removed. In the case of face-on disk motion, this mechanism would remove grains smaller than $\sim 1 \mu \mathrm{m}$ at all distances. Overall, it appears likely that any dust around RX J0806.4-4123 consists of predominantly large, $a>1 \mu \mathrm{m}$, grains - if the Herschel emission indeed comes from a dusty torus around the XTINS.

\section{CONCLUSIONS}

Using Herschel PACS, we detected $160 \mu \mathrm{m}$ emission close to positions of two out of the eight investigated NSs. Herschel PACS $70 \mu \mathrm{m}$, WISE and Spitzer IRAC observations resulted only in upper flux limits for the positions of the eight NSs. The ratios of the $160 \mu \mathrm{m}$ band luminosity to the bolometric luminosity of the respective XTINSs are between $5 \%$ and $20 \%$. If these detections are associated with the NSs, they would imply cold $\left(T_{d} \approx 20 \mathrm{~K}\right)$ dusty tori/belts around the NSs. For RX J0806.4-4123, the implied belt radius would be within the range discussed for fallback disks if only large $(a>10 \mu \mathrm{m})$ dust grains are present. The implied dust torus radius is larger for smaller grains (e.g., $r \approx 10^{15} \mathrm{~cm}$ for $\left.a=1 \mu \mathrm{m}\right)$, raising questions about the formation mechanism of such a dust belt. The higher X-ray luminosity of RX J2143.0+0654 would even imply a factor 3 larger torus radius. 
There is a $3 \%$ probability that both Herschel PACS red band detections are unrelated to the XTINSs, and there is a $33 \%$ probability that one of the two is unrelated to the respective XTINS. The relatively large offset of the faint Herschel emission in the case of RX J2143.0+0654 and the possibility of a galaxy counterpart suggest that this detection may be associated with a background galaxy. For RX J0806.4-4123, an interstellar cirrus is an alternative to the dusty-torus hypothesis. Deeper observations at shorter and longer wavelengths with better spatial resolution could probe whether or not the Herschel $160 \mu \mathrm{m}$ is associated with the NS.

For the six other NSs, we do not find any significant warm or cold dust emission. The reached flux ratio limits come close to those of bright debris disks around non-degenerate stars considering the warm dust, but for cold dust the Herschel observations are still not sensitive enough to exclude cold disks. In principle, our observations are still consistent with the presence of cold dusty disks around the NSs if one relies on the flux ratios found for other debris disks which are at least two orders of magnitudes smaller than what we achieved here. There is, of course, the other possibility that the XTINS do not harbor disks, either because they never had or because they lost them due to the composition of the dust in the disk (e.g., only small dust grains close to the NS). Currently, we cannot differentiate between the cold-disk and no-disk scenarios. Future submillimeter interferometer observations provide an opportunity to test whether or not cold disks are present around these fascinating NSs.

We would like to thank Hervé Bouy, Volker Tolls, and the staff from the NHSC Helpdesk for their helpful advice regarding Herschel data reduction. We would also like to thank Eliahu Dwek, András Gáspár, Paola Popesso, and Bruce Sibthorpe for detailed explanations and code sharing, and the referee for valueable comments.

This work is based on observations made with Herschel, a European Space Agency Cornerstone Mission with significant participation by NASA. Support for this work was provided by NASA through award RSA 1437567 issued by JPL/Caltech.

This work is based in part on observations made with the Spitzer Space Telescope, which is operated by the Jet Propulsion Laboratory, California Institute of Technology under a contract with NASA. Support for this work was provided by NASA through award RSA 1416202 issued by JPL/Caltech.

S.B.P. was supported by the grant RFBR 12-02-00186.

This publication makes use of data products from the Wide-field Infrared Survey Explorer, which is a joint project of the University of California, Los Angeles, and the Jet Propulsion Laboratory/California Institute of Technology, funded by the National Aeronautics and Space Administration.

\section{REFERENCES}

Alpar, M. A. 2001, ApJ, 554, 1245

-. 2007, Ap\&SS, 308, 133

Alpar, M. A., Ankay, A., \& Yazgan, E. 2001, ApJ, 557, L61
Backman, D. E., \& Paresce, F. 1993, in Protostars and Planets III, ed. E. H. Levy \& J. I. Lunine, 1253-1304

Berta, S., Magnelli, B., Nordon, R., et al. 2011, A\&A, 532, A49 Brown, M. E. 2001, AJ, 121, 2804

Bryden, G., Beichman, C. A., Trilling, D. E., et al. 2006, ApJ, 636, 1098

Çalişkan, Ş., Ertan, Ü., Alpar, M. A., Trümper, J. E., \& Kylafis, N. D. 2013, MNRAS, 431, 1136

Chatterjee, P., Hernquist, L., \& Narayan, R. 2000, ApJ, 534, 373

Chevalier, R. A. 1989, ApJ, 346, 847

Cordes, J. M., \& Lazio, T. J. W. 2002, ArXiv Astrophysics e-prints

Cutri, R. M., Wright, E. L., Conrow, T., et al. 2012, Explanatory Supplement to the WISE All-Sky Data Release Products, Tech. rep.

Debes, J. H., Walsh, K. J., \& Stark, C. 2012, ApJ, 747, 148

Dones, L., Weissman, P. R., Levison, H. F., \& Duncan, M. J. 2004, Oort cloud formation and dynamics, ed. G. W. Kronk, 153-174

Dorschner, J., Begemann, B., Henning, T., Jaeger, C., \& Mutschke, H. 1995, A\&A, 300, 503

Duncan, R. C., \& Thompson, C. 1992, ApJ, 392, L9

Dwek, E., \& Smith, R. K. 1996, ApJ, 459, 686

Eiroa, C., Marshall, J. P., Mora, A., et al. 2013, A\&A, 555, A11

Eisenbeiss, T., Ginski, C., Hohle, M. M., et al. 2010, Astronomische Nachrichten, 331, 243

Ekşi, K. Y., \& Alpar, M. A. 2005, ApJ, 620, 390

Ertan, Ü., \& Calışkan, Ş. 2006, ApJ, 649, L87

Ertan, Ü., Erkut, M. H., Ekşi, K. Y., \& Alpar, M. A. 2007, ApJ, 657,441

Fazio, G. G., Hora, J. L., Allen, L. E., et al. 2004, ApJS, 154, 10

Fruchter, A. S., \& Hook, R. N. 2002, PASP, 114, 144

Gáspár, A., \& Rieke, G. H. 2014, ApJ, 784, 33

Haberl, F. 2007, Ap\&SS, 308, 181

Haberl, F. 2013, in The Fast and the Furious: Energetic

Phenomena in Isolated Neutron Stars, Pulsar Wind Nebulae and Supernova Remnants, ed. J.-U. Ness

Haberl, F., Motch, C., Zavlin, V. E., et al. 2004, A\&A, 424, 635

Hambaryan, V., Hasinger, G., Schwope, A. D., \& Schulz, N. S. 2002, A\&A, 381, 98

Hobbs, G., Lorimer, D. R., Lyne, A. G., \& Kramer, M. 2005, MNRAS, 360, 974

Hobbs, G., Lyne, A. G., Kramer, M., Martin, C. E., \& Jordan, C. 2004, MNRAS, 353, 1311

Hohle, M. M., Haberl, F., Vink, J., et al. 2012, MNRAS, 423, 1194

Indebetouw, R., Mathis, J. S., Babler, B. L., et al. 2005, ApJ, 619,931

Jura, M., Farihi, J., \& Zuckerman, B. 2007, ApJ, 663, 1285

Kaplan, D. L., Chakrabarty, D., Wang, Z., \& Wachter, S. 2009, ApJ, 700, 149

Kaplan, D. L., Kamble, A., van Kerkwijk, M. H., \& Ho, W. C. G. 2011, ApJ, 736, 117

Kaplan, D. L., Kulkarni, S. R., \& van Kerkwijk, M. H. 2002, ApJ, 579, L29

-. 2003, ApJ, 588, L33

Kaplan, D. L., \& van Kerkwijk, M. H. 2009, ApJ, 705, 798

Kaspi, V. M. 2010, Proceedings of the National Academy of Science, 107, 7147

Lallement, R., Vergely, J.-L., Valette, B., et al. 2014, A\&A, 561, A91

Lutz, D., Poglitsch, A., Altieri, B., et al. 2011, A\&A, 532, A90

Michel, F. C., \& Dessler, A. J. 1981, ApJ, 251, 654

Mignani, R. P., Motch, C., Haberl, F., et al. 2009, A\&A, 505, 707

Morbidelli, A., Levison, H. F., \& Gomes, R. 2008, The Dynamical Structure of the Kuiper Belt and Its Primordial Origin, ed. M. A. Barucci, H. Boehnhardt, D. P. Cruikshank, A. Morbidelli, \& R. Dotson, 275-292

Motch, C., Pires, A. M., Haberl, F., Schwope, A., \& Zavlin, V. E. 2009, A\&A, 497, 423

Motch, C., Sekiguchi, K., Haberl, F., et al. 2005, A\&A, 429, 257

Olausen, S. A., \& Kaspi, V. M. 2013, ArXiv e-prints

Olausen, S. A., Zhu, W. W., Vogel, J. K., et al. 2013, ApJ, 764, 1

Perna, R., Duffell, P., Cantiello, M., \& MacFadyen, A. I. 2014, ApJ, 781, 119

Phillips, J. A., \& Chandler, C. J. 1994, ApJ, 420, L83

Pinte, C., \& Laibe, G. 2014, ArXiv e-prints 
Poglitsch, A., Waelkens, C., Geis, N., et al. 2010, A\&A, 518, L2 Pons, J. A., \& Geppert, U. 2007, A\&A, 470, 303

Pons, J. A., Miralles, J. A., \& Geppert, U. 2009, A\&A, 496, 207 Popesso, P., Magnelli, B., Buttiglione, S., et al. 2012, ArXiv e-prints

Popov, S. B., Pons, J. A., Miralles, J. A., Boldin, P. A., \& Posselt, B. 2010, MNRAS, 401, 2675

Posselt, B., Neuhäuser, R., \& Haberl, F. 2009, A\&A, 496, 533

Posselt, B., Popov, S. B., Haberl, F., et al. 2007, Ap\&SS, 308, 171

Posselt, B., Schreyer, K., Perna, R., et al. 2010, MNRAS, 405, 1840

Reach, W. T., Megeath, S. T., Cohen, M., et al. 2005, PASP, 117, 978

Schwope, A. D., Erben, T., Kohnert, J., et al. 2009, A\&A, 499, 267

Seki, J., \& Yamamoto, T. 1980, Ap\&SS, 72, 79

Sibthorpe, B., Ivison, R. J., Massey, R. J., et al. 2013, MNRAS, 428, L6

Skrutskie, M. F., Cutri, R. M., Stiening, R., et al. 2006, AJ, 131, 1163

Su, K. Y. L., Chu, Y.-H., Rieke, G. H., et al. 2007, ApJ, 657, L41

Thompson, C., Lyutikov, M., \& Kulkarni, S. R. 2002, ApJ, 574, 332
Trümper, J. E., Dennerl, K., Kylafis, N. D., Ertan, Ü., \& Zezas, A. 2013, ApJ, 764, 49

Trümper, J. E., Zezas, A., Ertan, Ü., \& Kylafis, N. D. 2010, A\&A, 518, A46

van Kerkwijk, M. H., Kaplan, D. L., Pavlov, G. G., \& Mori, K. 2007, ApJ, 659, L149

van Paradijs, J., Taam, R. E., \& van den Heuvel, E. P. J. 1995, A\&A, 299, L41

Veneziani, M., Piacentini, F., Noriega-Crespo, A., et al. 2013 ApJ, 772, 56

Viganò, D., \& Pons, J. A. 2012, MNRAS, 425, 2487

Vuong, M. H., Montmerle, T., Grosso, N., et al. 2003, A\&A, 408, 581

Walter, F. M., Eisenbeiß, T., Lattimer, J. M., et al. 2010, ApJ, 724,669

Wang, Z. 2014, ArXiv e-prints

Wang, Z., Chakrabarty, D., \& Kaplan, D. L. 2006, Nature, 440, 772

Wang, Z., Kaplan, D. L., \& Chakrabarty, D. 2007a, ApJ, 655, 261 Wang, Z., Kaspi, V. M., \& Higdon, S. J. U. 2007b, ApJ, 665, 1292 Werner, M. W., Roellig, T. L., Low, F. J., et al. 2004, ApJS, 154,

Wright, E. L., Eisenhardt, P. R. M., Mainzer, A. K., et al. 2010, AJ, 140,1868

Wyatt, M. C. 2008, ARA\&A, 46, 339

APPENDIX

\section{A. ADDITIONAL RESULTS ON INDIVIDUAL SOURCES}

The following subsections present detailed results from the WISE, Spitzer and Herschel observations for the NSs without any significant IR detections. In contrast to Table 5, no color correction has been applied to the quoted flux limits. The Figures have different (manually tweaked) color scales and smoothing (only in the case of Spitzer; usually gaussian with a kernel radius of 3 pixel), optimized to emphasize faint fluxes in the surrounding of the NS position. Circles in the pictures do not represent error circles. In the case of Spitzer, $r=2$ "' 4 (2 native IRAC pixel), the circle has the size of the used photometry aperture. In the case of Herschel and WISE, $r=10^{\prime \prime}$, they merely indicate the NS position. For an overview about the instrumental FWHM in each individual observing band, we refer to Sections 3 and 4.3. Position uncertainties of the targets and instrumental pointing errors are discussed in Section 3 as well as below if necessary.

\section{A.1. RX J0420.0-5022}

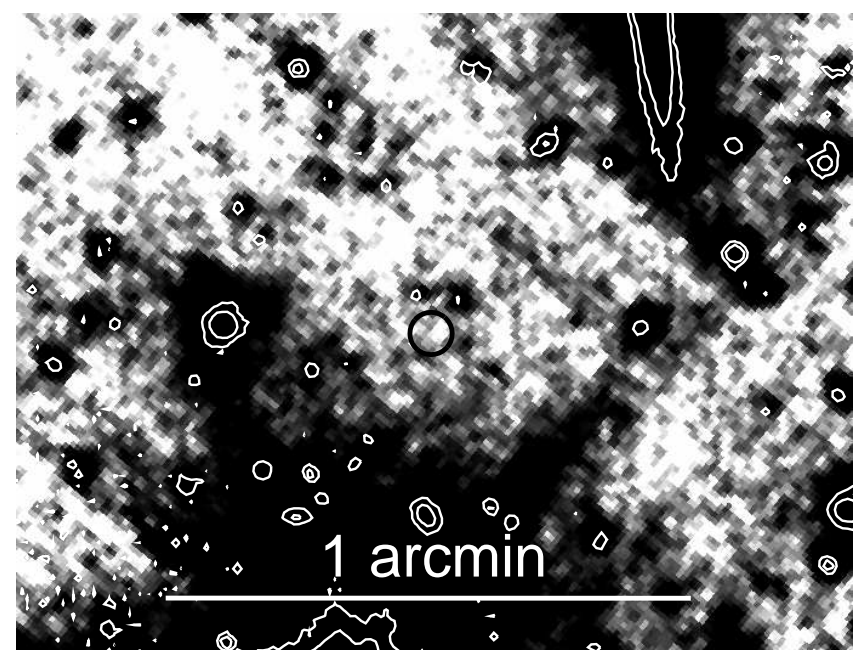

Figure A1. Spitzer IRAC2 $(4.5 \mu \mathrm{m})$ map around RX J0420.0-5022. The image is $1 ! 6 \times 1 ! 2$, North is up, East to the left. White contours are from our previous VLT $H$-band observations (Posselt et al. 2009). The INS position is marked with a black circle with a radius of 2 native IRAC pixels (4 image pixels, corresponding to 2"'4). Note that the astrometric uncertainty for the expected RX J0420.0-5022 position is less than $2^{\prime \prime}$, according to constraints by Mignani et al. (2009).

There is no IR emission at the position of RX J0420.0-5022 in the Spitzer 4.5 $\mu \mathrm{m}$ IRAC image, see Figure A1 The aperture corrected $5 \sigma$ upper limit is $F_{5 \sigma}^{4.5 \mu \mathrm{m}}=2.8 \mu \mathrm{Jy}$. 


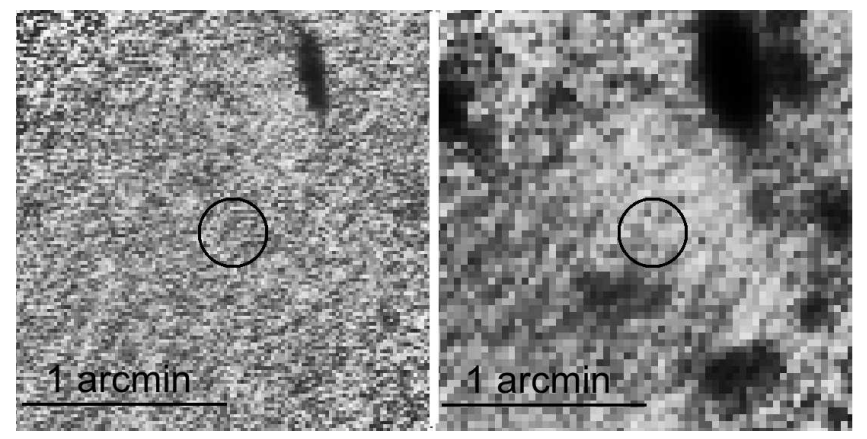

Figure A2. Herschel PACS maps around RX J0420.0-5022. The blue (60-85 $\mu \mathrm{m})$ and red (130-210 $\mu \mathrm{m})$ bands are shown in the left and right panels, respectively. Each map is $2^{\prime} \times 2^{\prime}$, North is up, East is to the left. The circle with a radius of $10^{\prime \prime}$ shows the NS position.

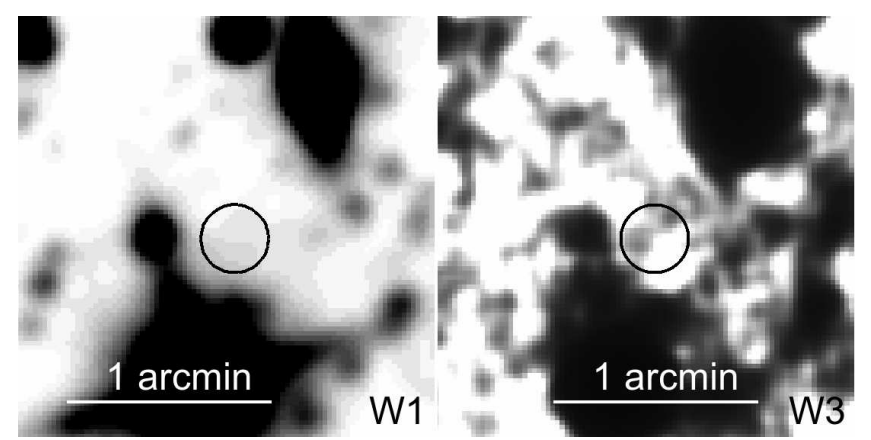

Figure A3. WISE W1 $(3.6 \mu \mathrm{m})$ and W3 $(12 \mu \mathrm{m})$ maps around the position of RX J0420.0-5022. The same field of view is shown as in the Herschel PACS maps of Figure A2

At the position of RX J0420.0-5022, we detect no source in the Herschel blue and red band (see Figure A2). Using apertures in different source-free regions and at the target position, we determined the $5 \sigma$ upper flux limits at the INS position as $F_{5 \sigma}^{\text {blue }}=4.5 \mathrm{mJy}$ and $F_{5 \sigma}^{\mathrm{red}}=7 \mathrm{mJy}$. There are several common sources in the Herschel bands and the WISE bands W1 to W3. There is no noticeable astrometric shift between these bands. The W1 and W3 images for the field around RX J0420.0-5022 are shown in Figure A3. There is very faint emission east and northwest of the INS in the W3 band, but no entry in the WISE source catalog at these positions. Slightly enhanced emission at the same positions might be suspected in the red band (Figure A2). However, these potential W3 sources are separated at least $5{ }^{\prime \prime} 8$ from the expected INS position and are therefore unassociated to the INS.

\section{A.2. $R X J 0720.4-3125$}
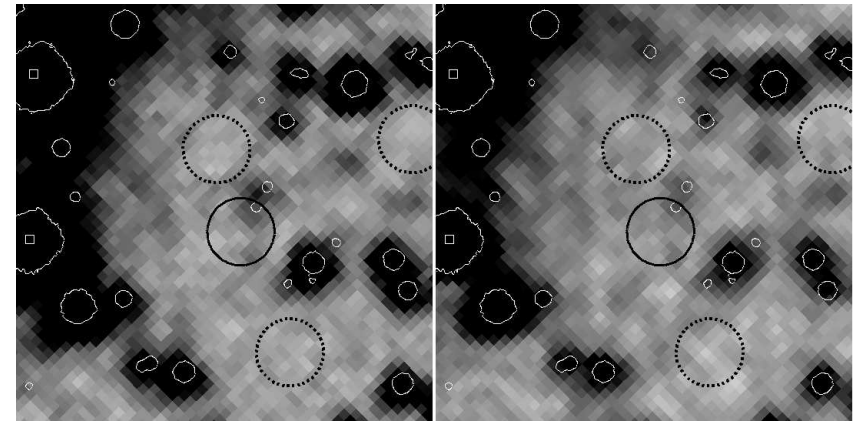

Figure A4. Spitzer $3.6 \mu \mathrm{m}$ (left) and $4.5 \mu \mathrm{m}$ (right) IRAC mosaic around RX J0720.4-3125. Each map is $30^{\prime \prime} \times 30^{\prime \prime}$, North is up, East is to the left. The overlaid white contours are from the VLT ISAAC $H$-band image (Posselt et al. 2009), the small circles in two eastern sources mark 2MASS point source positions. The black circle with radius of 2'! 4 (2 native IRAC pixels) marks the position of RX J0720.4-3125 at the time of the Spitzer observations. The dashed circles indicate three examples of the ten apertures used to determine the background and noise in source-free image regions.

We first consider the Spitzer observations. The IRAC mosaics are very crowded, and there appears to be a faint source close to the position of RX J0720.4-3125 (Figure A4). The expected position of the INS at the time of the Spitzer observation, MJD 55582, is $\alpha=7^{h} 20^{m} 24.904 ; \delta=-31^{\circ} 25^{\prime} 49^{\prime \prime}$.63. It is calculated using the latest, MJD $=54477$, optical position of the NS and its proper motion, $\mu_{\alpha}=-93.2 \pm 5.4$ mas yr $^{-1}$ and $\mu_{\delta}=48.6 \pm 5.1$ mas yr $^{-1}$, listed by 
Eisenbeiss et al. (2010). The radial positional uncertainty of the expected INS position at the time of the Spitzer observation is 62 mas (90\% confidence level). The $1 \sigma$ astrometric accuracy of the $3.6 \mu \mathrm{m}$ and $4.5 \mu \mathrm{m}$ mosaics is $0^{\prime \prime} 27$ and $0 . \prime 28$, respectively (Section 4.2). The faint IRAC $3.6 \mu \mathrm{m}$ source closest to RX J0720.4-3125 seems to be a blended source and has a spatial separation of at least 1"3. From our previous VLT ISAAC $H$-band observations (Posselt et al. 2009) two individual NIR sources are known close to the position of the faint IRAC source(s). From the spatial separation and the NIR identification of the faint IRAC source(s) we conclude that the IRAC source is not the counterpart of the INS. Thus, RX J0720.4-3125 is undetected at $3.6 \mu \mathrm{m}$ and $4.5 \mu \mathrm{m}$.

To obtain an upper limit, we determine the background level and the standard deviation of 10 source-free apertures close to the source position. We use the residual mosaics (Section 4.2) for these measurements. We chose apex-qa task parameters which result in a good removal of point sources in the immediate surrounding of the INS. The northern part of the faint blended source close to the INS is detected as individual point source and removed in both IRAC channels. A small flux enhancement remains in both channels at the position of the southern ISAAC source and slightly increases the derived upper limits, which are computed by the measured source aperture flux above the background plus five times the standard deviation of the 10 source-free apertures (Section 4.2). The aperture-corrected $3.6 \mu \mathrm{m}$ and the $4.5 \mu \mathrm{m}$ upper flux limits at the position of RX J0720.4-3125 are $F_{5 \sigma}^{3.6 \mu \mathrm{m}}=4.9 \mu \mathrm{Jy}$ and $F_{5 \sigma}^{4.5 \mu \mathrm{m}}=4.5 \mu \mathrm{Jy}$, respectively.

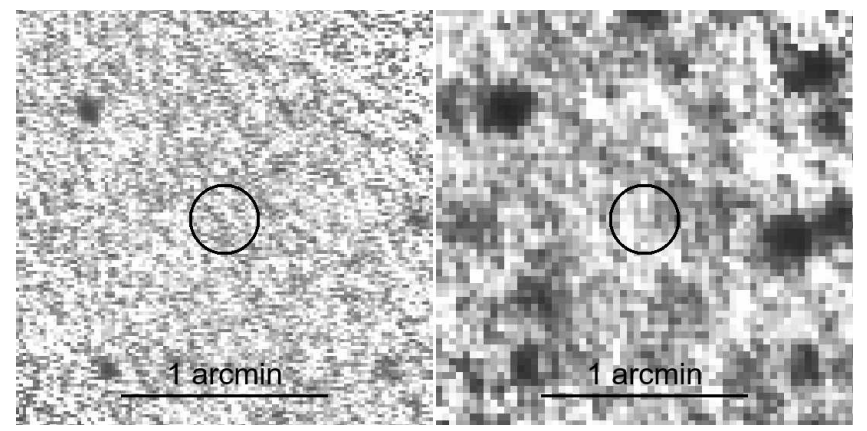

Figure A5. Herschel PACS maps around RX J0720.4-3125. The blue (60-85 $\mu \mathrm{m})$ and red $(130-210 \mu \mathrm{m})$ bands are shown in the left and right panels, respectively. Each map is $2^{\prime} \times 2^{\prime}$, North is up, East is to the left. The circle with a radius of $10^{\prime \prime}$ shows the XTINS position.

There is no obvious infrared source in the Herschel blue and red band at the position of RX J0720.4-3125 (see Figure A5). The closest possible source in the red band is at least $6^{\prime \prime}$ from the INS position. Using apertures in different source-free regions and at the target position, we determine the $5 \sigma$ upper flux limits for the INS to be $F_{5 \sigma}^{\text {blue }}=5.2 \mathrm{mJy}$ and $F_{5 \sigma}^{\text {red }}=9.4 \mathrm{mJy}$.

The spatial resolution and sensitivity of the two Spitzer IRAC images are superior to the first two channels of the WISE data, but the W3 and W4 band provide new wavelength coverage. Nothing is seen in W3 or W4 at the position of the INS. Because of the uncertainty in the W4 astrometry, only W3 is shown in Figure A6. The closest, very faint $12 \mu \mathrm{m}$ source in the east of the INS has a spatial separation from the INS of $\sim 5^{\prime \prime}$. Given the good astrometric compliance of major W3 sources with Spitzer IRAC sources, this faint source is unlikely to be the INS counterpart.

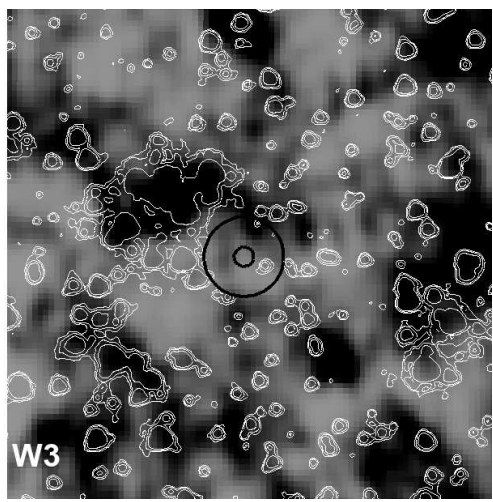

Figure A6. W3 $(12 \mu \mathrm{m})$ map around the position of RX J0720.4-3125. The field of view is $2^{\prime} \times 2^{\prime}$, north is up, east is to the left. The white overlay contours are the Spitzer $4.5 \mu \mathrm{m}$ IRAC contours. The black circles mark the position of RX J0720.4-3125, the small one is the same as indicated in the Spitzer image (Fig. A4 radius 2!'4), the larger one has a radius of $10^{\prime \prime}$ as in the Herschel PACS maps (Fig. A5). 


\section{A.3. $R X J 1308.6+2127$}

There is no IR emission at the position of RX J1308.6+2127 in the Spitzer 4.5 $\mu \mathrm{m}$ IRAC image, see Figure A8 The aperture corrected $5 \sigma$ upper limit is $F_{5 \sigma}^{4.5 \mu \mathrm{m}}=1.6 \mu \mathrm{Jy}$.

In the Herschel PACS blue band, there is no significant emission around the position of RX J1308.6+2127, see Figure A7 Using apertures in different source-free regions and at the target position we determine the $5 \sigma$ upper flux limit for the INS as $F_{5 \sigma}^{\text {blue }}=1.7 \mathrm{mJy}$. In the Herschel PACS red band, there is no significant emission at the position of RX J1308.6+2127, but there is faint emission southwest and west of it (Figure A7). The spatial separation of this faint emission and the XTINS position is $10^{\prime \prime}$. The positional uncertainty of the NS at the $90 \%$ confidence level in the Herschel observation is smaller than $0^{\prime \prime} .7$ with account of the uncertainty of the Chandra position (Kaplan et al. 2002; Hambaryan et al. 2002) and the uncertainty of the proper motion (Motch et al.|2009). We checked for common sources in the Herschel observation, 2MASS point source catalog and Spitzer IRAC $4.5 \mu \mathrm{m}$ data. Based on this comparison, we confirm that the absolute Herschel pointing accuracy is better than $1^{\prime \prime} .5$ for this observation as expected from the works by the Herschel calibration and HIPE teams (see above). Thus, all the faint sources in the Herschel PACS red band can be excluded as INS counterparts. Using apertures in different source-free regions and at the target position, we determine the $5 \sigma$ upper flux limit in the red band for the INS as $F_{5 \sigma}^{\text {red }}=5.2 \mathrm{mJy}$.

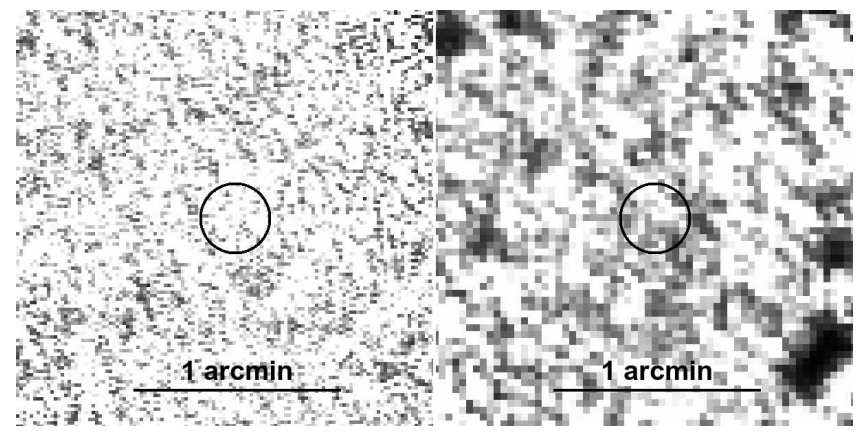

Figure A7. Herschel PACS maps around RX J1308.6+2127. The blue $(60-85 \mu \mathrm{m})$ and red $(130-210 \mu \mathrm{m})$ bands are shown in the left and right panels, respectively. Each map is $2^{\prime} \times 2^{\prime}$, North is up, East is to the left. The circle with a radius of $10^{\prime \prime}$ shows the NS position.

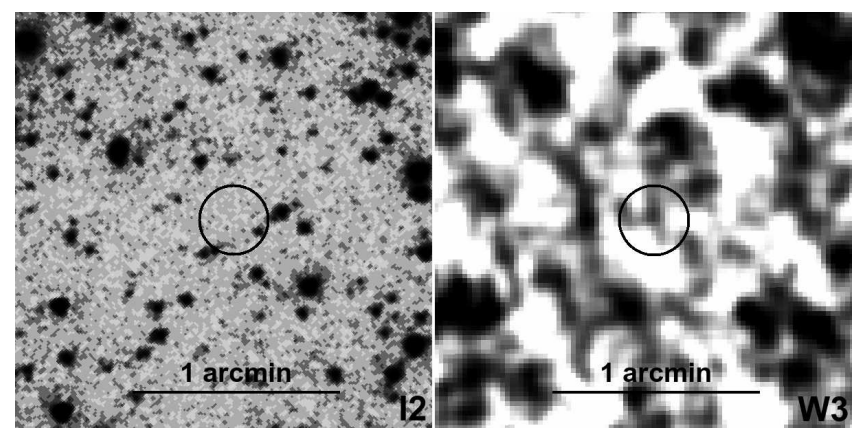

Figure A8. Spitzer IRAC2 $(4.5 \mu \mathrm{m}$, left) and WISE W3 $(12 \mu \mathrm{m}$, right) maps around the position of RX J1308.6+2127. The same field of view is shown as in the Herschel PACS maps of Figure A7

We compared the WISE W1-W3 data to the Spitzer IRAC $4.5 \mu \mathrm{m}$ data. There is no noticeable astrometric shift between Spitzer IRAC $4.5 \mu \mathrm{m}$ and WISE W1 and W2. For WISE W3 there are only few clear common sources, three of them are 2MASS point sources. There is no noticeable astrometric shift for these sources. Interestingly, there is faint emission in WISE W3 at a position consistent with the INS position within positional accuracy, see Figure A8. There is no source reported in the WISE All-Sky Source Catalog at this position, and no such emission is seen in any other WISE band, the Spitzer IRAC $4.5 \mu \mathrm{m}$ or the Herschel PACS bands. Due to its faintness the W3 emission can be a noise feature. Confirmation by similar observations are necessary before any conclusions regarding the INS can be drawn. In principle, emission at such wavelengths is particularly interesting, because - if real - it could indicate potential silicate emission in the $8-12 \mu \mathrm{m}$ region reminiscent of white dwarf debris disks (e.g., in the case of the spectacularly debris-polluted white dwarf GD 362, one-third of the debris disk emission is carried by a strong $10 \mu \mathrm{m}$ silicate emission feature; Jura et al. 2007). 


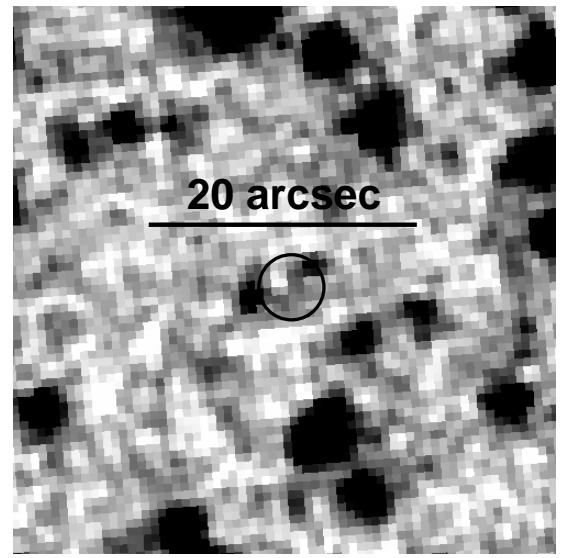

Figure A9. Spitzer IRAC2 $(4.5 \mu \mathrm{m})$ map around RX J1605.3+3249 $\left(40^{\prime \prime} \times 40^{\prime \prime}\right.$, North is up, East is to the left). The circle with a radius of $2.4^{\prime \prime}$ shows the expected INS position at the time of the Spitzer observations.

\section{A.4. $R X J 1605.3+3249$}

There is very faint IR emission at the position of RX J1605.3+3249 in the Spitzer 4.5 $\mu$ m IRAC image, see Figure A9, However, the INS position is located between two IR sources whose PRFs affect any measured flux at the INS position. We found that the faint $4.5 \mu \mathrm{m}$ emission has a significance of only $1.3 \sigma$ if we employ PRF subtraction of the two sources in the vicinity $(2.9 \sigma$ otherwise). Hence, there is no significant emission at $4.5 \mu \mathrm{m}$ from the INS. The aperture corrected $5 \sigma$ upper limit is $F_{5 \sigma}^{4.5 \mu \mathrm{m}}=3.6 \mu \mathrm{Jy}$.

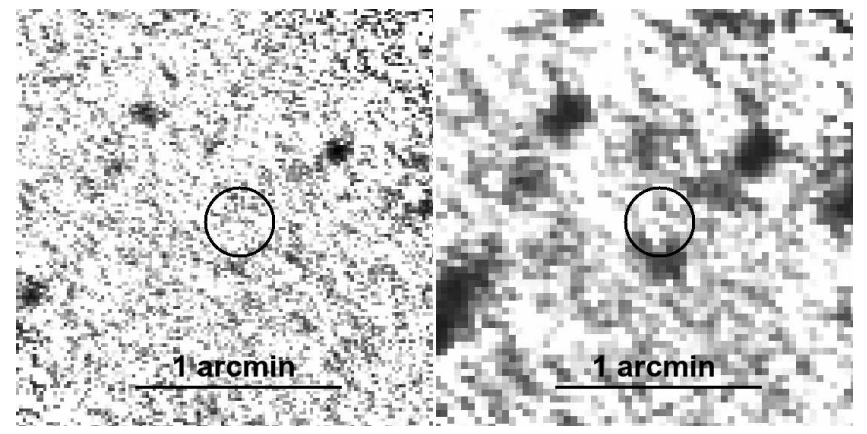

Figure A10. Herschel PACS maps around RX J1605.3+3249. The blue $(60-85 \mu \mathrm{m})$ and red $(130-210 \mu \mathrm{m})$ bands are shown in the left and right panels, respectively. Each map is $2^{\prime} \times 2^{\prime}$, North is up, East is to the left. The circle with a radius of $10^{\prime \prime}$ shows the NS position.

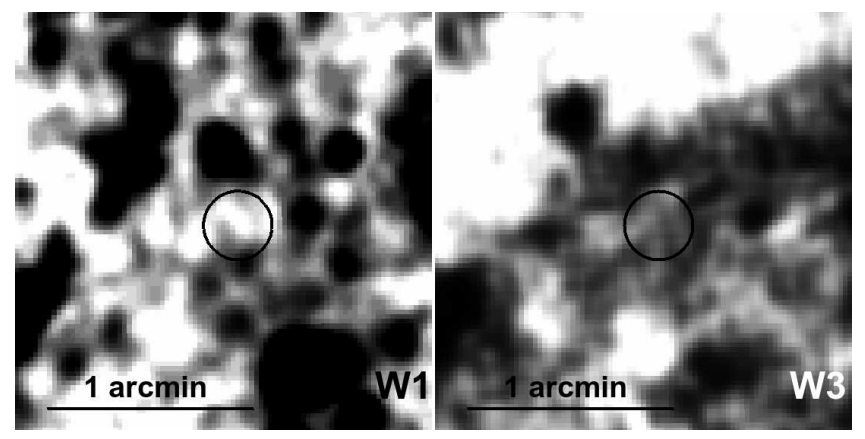

Figure A11. WISE W1 $(3.6 \mu \mathrm{m})$ and W3 $(12 \mu \mathrm{m})$ maps around the position of RX J1605.3+3249. The same field of view is shown as in the Herschel PACS maps of Figure A10

There are several common sources in the Herschel bands and the WISE bands W1 to W3. There is no noticeable astrometric shift between these bands. There is no source in the Herschel blue and red bands at the position of RX J1605.3+3249 (see Figure A10). North of the INS there is a faint source in the red band which is at least 5".6 separated from the expected INS position and is therefore unassociated to the INS. Using apertures in different sourcefree regions and at the target position, we determine the $5 \sigma$ upper limits as $F_{5 \sigma}^{\text {blue }}=6.1 \mathrm{mJy}$ and $F_{5 \sigma}^{\text {red }}=12.2 \mathrm{mJy}$. 
The WISE W1 and W3 images for the field around RX J1605.3+3249 are shown in Figure A11, While there is large scale extended emission in W3, no point source is seen at position of RX J1605.3+3249 in any of the WISE bands.

\section{A.5. $R X J 1856.5-3754$}

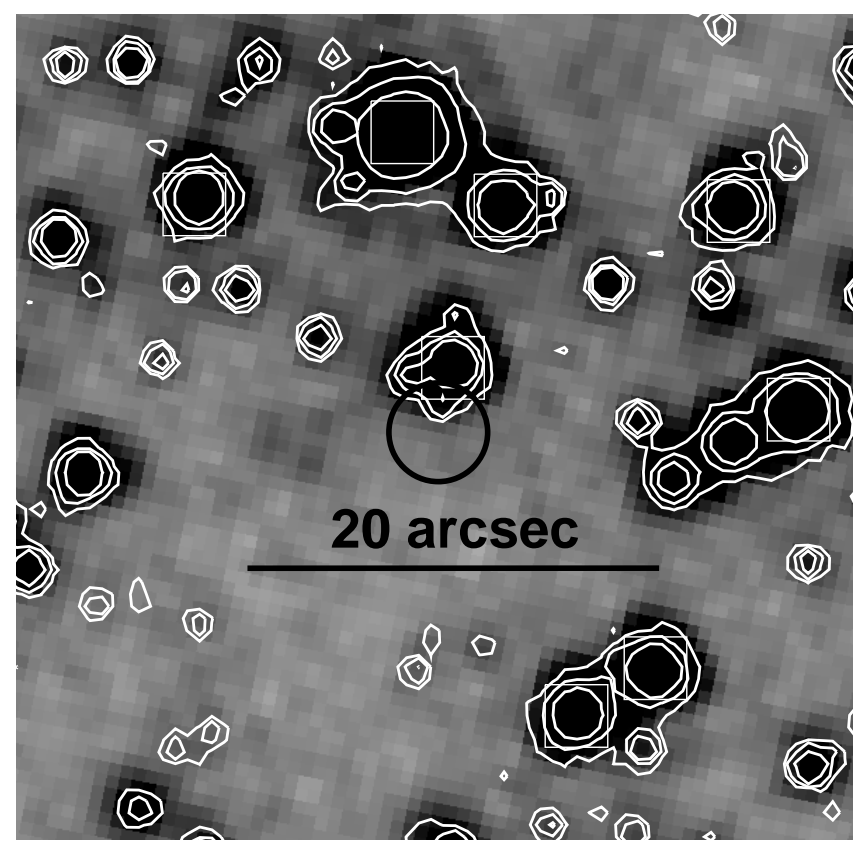

Figure A12. Spitzer IRAC2 $(4.5 \mu \mathrm{m})$ map around RX J1856.5-3754. The image is $40^{\prime \prime} \times 40^{\prime \prime}$, North is up, East is to the left. The circle with a radius of $2.4^{\prime \prime}$ shows the expected INS position at the time of the Spitzer observations. The overplotted white contours are from the VLT ISAAC $H$-band image (Posselt et al. 2009), the white boxes indicate 2MASS point sources.

The positions of RXJ1856.5-3754 at the time of the Spitzer and Herschel observations were estimated from the position and proper motion values listed by Walter et al. (2010). The accuracy of the determined positions are better than $0{ }^{\prime \prime} 1$. The astrometry of the Spitzer IRAC2 $(4.5 \mu \mathrm{m})$ image of RX J1856.5-3754 is well aligned with the astrometry of previous $H$-band observations (Figure $\mathrm{A12}$ ). There is no prominent emission centered at the expected INS position, but there is strong IR emission north of RX J1856.5-3754, starting at $\approx 1^{\prime \prime}$ from the INS. From the NIR we know this source consists of at least four individual sources which are, however, not discernable with Spitzer. We were not able to completely remove the IR emission of these northern sources by PRF fitting. Thus, our subsequently derived aperture-corrected $5 \sigma$ upper limit for RX J1856.5-3754, $F_{5 \sigma}^{4.5 \mu \mathrm{m}}=11.6 \mu \mathrm{Jy}$, is a very conservative estimate.

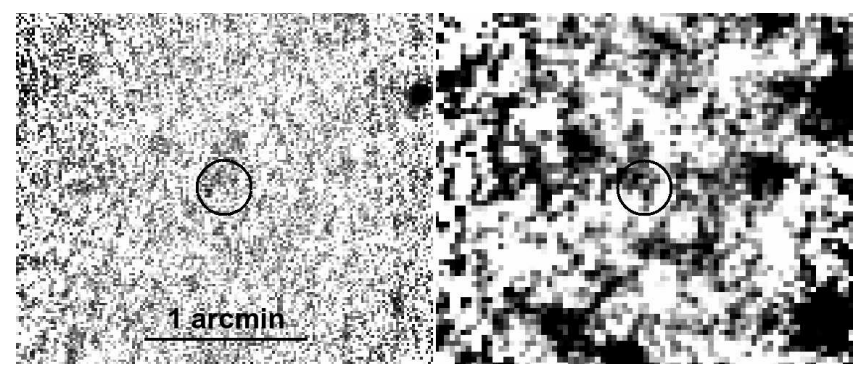

Figure A13. Herschel PACS maps around RX J1856.5-3754. The blue $(60-85 \mu \mathrm{m})$ and red (130-210 $\mu \mathrm{m})$ bands are shown in the left and right panels, respectively. North is up, East is to the left. The circle with a radius of $10^{\prime \prime}$ shows the NS position.

Comparing Herschel PACS and WISE sources we were not able to identify enough unique common sources to check the astrometry of the Herschel observations. Therefore, we assume the astrometric error to be the absolute pointing error of $\approx 2$ ". 4 . This value was listed by the Herschel calibration team as average value for a time window which includes our observation 18 . In the Herschel blue band, there is very faint emission at $\approx 44^{\prime \prime} 6$ north-east from the INS position. In the Herschel red band, the INS position is located within a larger faint emission region which probably

18 herschel.esac.esa.int/twiki/bin/view/Public/SummaryPointing 
consists of several sources and extends to the north-east and north (see Figure A13). We cannot exclude that the INS contributes to the faint PACS $160 \mu \mathrm{m}$ band emission. However, it appears likely that most of that emission actually comes from the same NIR sources which are also detected by Spitzer. We measured the flux at the INS position in an aperture with radius $5^{\prime \prime}$. The aperture-corrected flux is $F^{\text {red }}=3.1 \mathrm{mJy}$, which corresponds to $\approx 3 \sigma$ above the background. The nominal $5 \sigma$ flux limit for the position of RX J1856.5-3754 in the red band is $F_{5 \sigma}^{\mathrm{red}}=7.8 \mathrm{mJy}$. In the blue band, we used apertures in different source-free regions and at the target position to determine the $5 \sigma$ upper flux limit at the INS position as $F_{5 \sigma}^{\text {blue }}=7.7 \mathrm{mJy}$.

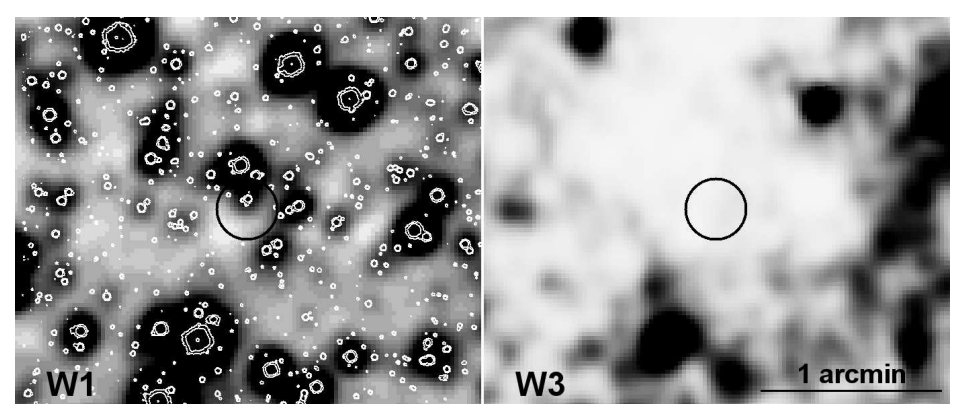

Figure A14. WISE W1 $(3.6 \mu \mathrm{m})$ and W3 $(12 \mu \mathrm{m})$ maps around the position of RX J1856.5-3754. The same field of view is shown as in the Herschel PACS maps of Figure A13 The white contours in the W1 image are from the VLT ISAAC $H$-band image (Posselt et al. 2009).

Comparing sources in the WISE W1-W3 bands images with $H$-band sources from a deep VLT observation (Posselt et al. 2009), there is no apparent astrometric shift between these bands (see Figure A14). The IR sources observed with W1 and W2 correspond to the ones seen with Spitzer IRAC $4.5 \mu \mathrm{m}$. Nothing is detected around the INS position in W3.

\section{A.6. PSR J1848-1952}

The position of PSR J1848-1952 at MJD 48695 is known with a $2 \sigma$ accuracy of $0^{\prime \prime} 6$ in right ascension, but $7^{\prime \prime}$ in declination (Hobbs et al. 2004). The proper motion is unknown for this pulsar. PSR J1848-1952 was recently observed for $38 \mathrm{ksec}$ with XMM-Newton (obsid:0653300101, PI Kaspi). Unfortunately, the pulsar was not detected (Olausen et al. 2013), the closest X-ray source to the pulsar radio position has an angular separation of $\approx 1$.'65. Thus, the X-ray observations cannot be used to determine the current position of PSR J1848-1952 with higher accuracy. Hobbs et al. (2004) gave the following proper motion values $\mu_{\alpha} \cos \delta=39(157) \mathrm{mas} \mathrm{yr}^{-1}$ and $\mu_{\delta}=-1200(1900)$ mas $\mathrm{yr}^{-1}$. While the error in declination is too large to be used for a reasonable upper limit on the expected pulsar proper motion, we use the error in right ascension to determine the maximal angular separation of the possible pulsar position in this direction at the time of the Herschel observation as $33^{\prime \prime} 1(2 \sigma)$. The mean 2 D speed of pulsars is $\approx 250 \mathrm{~km} \mathrm{~s}^{-1}$, and PSR B2224+64 has the highest inferred 2D speed of $\approx 1600 \mathrm{~km} \mathrm{~s}^{-1}$ (Hobbs et al. 2005). We assume this most extreme case to determine the maximal expected shift in declination for PSR J1848-1952. Its DM $=18.23 \mathrm{~cm}^{-3} \mathrm{pc}$ translates into a distance of $750 \mathrm{pc}$ using the NE2001 model for the Galactic distribution of free electrons (Cordes \& Lazio 2002). We estimate the upper limit of the proper motion of PSR J1848-1952 as 0 "' $45 \mathrm{yr}^{-1}$. Thus, at the time of the Herschel observation, the maximal expected shift is $9^{\prime \prime}$. We assume this value as upper limit on the proper motion in the direction of declination.

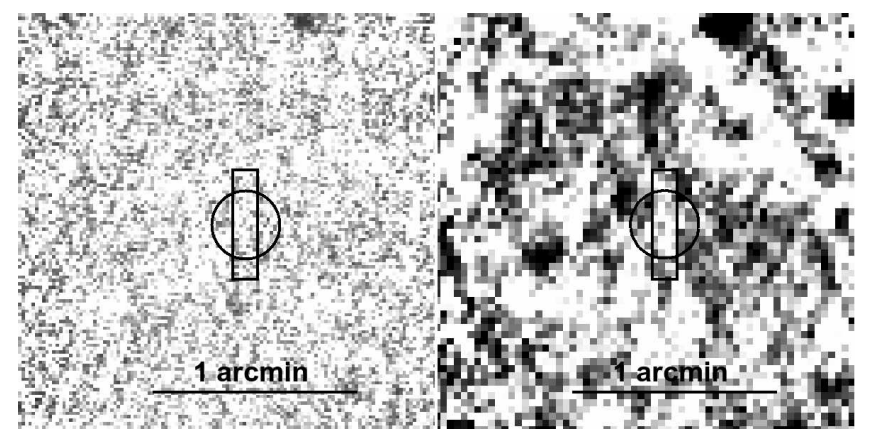

Figure A15. Herschel PACS maps around PSR J1848-1952. The blue $(60-85 \mu \mathrm{m})$ and red $(130-210 \mu \mathrm{m})$ bands are shown in the left and right panels, respectively. Each map is $2^{\prime} \times 2^{\prime}$, North is up, East is to the left. The circle with a radius of $10^{\prime \prime}$ marks the pulsar position, the $7 !^{\prime \prime} 4 \times 32^{\prime \prime}$ box shows the upper limits of the unknown proper motion together with the radio position uncertainty (see text). 


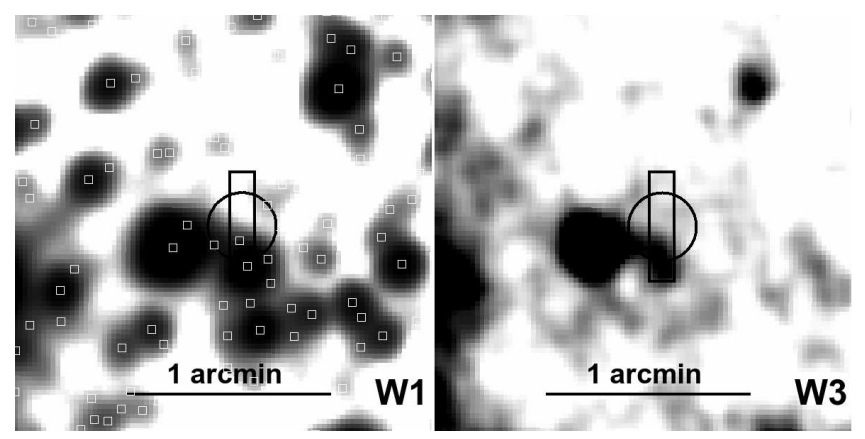

Figure A16. WISE W1 $(3.6 \mu \mathrm{m})$ and W3 $(12 \mu \mathrm{m})$ maps around the position of PSR J1848-1952. The same field of view is shown as in the Herschel PACS maps of Figure A15 The white boxes in W1 mark position of 2MASS point sources. The circle with a radius of $10^{\prime \prime}$ marks the pulsar position, the $7{ }^{\prime \prime} 4 \times 32^{\prime \prime}$ box shows the upper limits of the unknown proper motion together with the radio position uncertainty (see text).

The sky region around PSR J1848-1952 is densly populated with infrared sources. Comparing 2MASS, WISE and our Herschel observation, it is difficult to pin down common sources. However, we found at least three common sources between these bands. From the comparison of their positions the expected absolute pointing error of 1". 1 for the Herschel observation seems to be a reasonable estimate. There is no source in the Herschel blue and red band at the position of RX J1848-1952 (see Figure A15). There is a faint southern source which is inside the box of proper motion upper limits. However this source has a counterpart in other bands, in particular in 2MASS. Therefore, it is not the counterpart of the pulsar. Using apertures in different source-free regions and at the target position, we determine the $5 \sigma$ upper flux limits at the INS position as $F_{5 \sigma}^{\text {blue }}=3.6 \mathrm{mJy}$ and $F_{5 \sigma}^{\text {red }}=5.9 \mathrm{mJy}$.

The WISE W1 and W3 images for the field around PSR J1848-1952 are shown in Figure A16. Except for the southern 2MASS source, there is no WISE point source deteced in the region of PSR J1848-1952.

\section{B. TEMPERATURE DISTRIBUTION OF DUST AROUND A NEUTRON STAR}

We know from X-ray and UV observations of XTINSs that their radiation can be approximately described as blackbody radiation with temperatures $k T_{\mathrm{NS}}=40-100 \mathrm{eV}$ (i.e., $T_{\mathrm{NS}}=0.5-1.2 \times 10^{6} \mathrm{~K}$; see, e.g., Haberl 2007). This radiation heats the dust grains around a XTINS. If collisions of a grain with surrounding particles can be neglected, its temperature $T_{\mathrm{g}}$ is determined by the balance between the radiative heating and cooling (e.g., Backman \& Paresce 1993):

$$
\left(R_{\mathrm{NS}} / r\right)^{2} \int_{0}^{\infty} \epsilon_{\nu}^{\mathrm{abs}}(a) \pi B_{\nu}\left(T_{\mathrm{NS}}\right) d \nu=4 \int_{0}^{\infty} \epsilon_{\nu}^{\mathrm{em}}(a) \pi B_{\nu}\left(T_{\mathrm{g}}\right) d \nu,
$$

where $R_{\mathrm{NS}}$ is the stellar radius, $r$ is the distance of the spherical grain with radius $a$ to the NS, $B_{\nu}(T)$ is the Planck function in dependence on the NS surface temperature, $T_{\mathrm{NS}}$, or the grain temperature $T_{\mathrm{g}}, \epsilon_{\nu}^{\mathrm{abs}}(a)$ and $\epsilon_{\nu}^{\mathrm{em}}(a)$ are the grain's absorption and emission efficiencies, which depend on the radiation wavelength (frequency), the grain size, and the properties of grain material.

If the grain radius $a$ is much larger than the wavelength of the heating radiation (e.g., $a \gg 0.03 \mu \mathrm{m}$ for $T_{\mathrm{NS}}=$ $\left.0.5 \times 10^{6} \mathrm{~K}\right)$, the absorption efficiency can be approximated as $\epsilon_{\nu}^{\text {abs }} \approx 1$, i.e., the grains are nearly perfect absorbers. For the UV and soft X-rays considered here, this approximation is consistent with the detailed calculations by, e.g., Dwek \& Smith (1996). At large distances from the XTINS we expect cold dust grains, which emit long-wavelength radiation and are inefficient emitters. In the Rayleigh limit, $\lambda \gg 2 \pi a$ ( or $\nu \ll c /(2 \pi a)$ ), the emission efficiency of a dielectric grain is (e.g., Seki \& Yamamoto 1980):

$$
\epsilon_{\nu}^{\mathrm{em}} \approx \frac{8 \pi a \nu}{c} \operatorname{Im}\left[\frac{\varepsilon_{\nu}-1}{\varepsilon_{\nu}+2}\right]=\frac{2 \pi a \nu}{c} \frac{24 n_{\nu} k_{\nu}}{\left(n_{\nu}^{2}-k_{\nu}^{2}+2\right)^{2}+4 n_{\nu}^{2} k_{\nu}^{2}} \ll 1
$$

where $\varepsilon_{\nu}=\left(n_{\nu}+i k_{\nu}\right)^{2}$ is the dielectric constant, and $k_{\nu}$ and $n_{\nu}$ are the imaginary and real parts of the complex refraction coefficient of the grain material. The quantities $n_{\nu}$ and $k_{\nu}$ depend on the grain's chemical composition, and their dependence on $\nu$ can be quite complex, including resonances at some frequencies/wavelengths (e.g., at $\lambda \approx 10$ and $\approx 20 \mu \mathrm{m}$ for silicate grains; see Dorschner et al. 1995). However, at wavelengths well above the resonance wavelength, $k_{\nu}$ and $n_{\nu}$ show a universal frequency dependence:

$$
n_{\nu}=n, \quad k_{\nu}=k_{0}\left(\nu / \tilde{\nu}_{0}\right) \ll n, \quad \text { at } \quad \nu<\tilde{\nu}_{0},
$$

where $\tilde{\nu}_{0}$ is a frequency below the lowest resonance frequency, and $k_{0}$ is a constant that depends on the grain composition. Using these expressions for $k_{\nu}$ and $n_{\nu}$, it is convenient to parameterize the long-wavelength emission efficiency as follows:

$$
\epsilon_{\nu}^{\mathrm{em}}=\epsilon_{0}\left(\nu / \nu_{0}\right)^{2}
$$


where

$$
\nu_{0}=\left(\frac{\tilde{\nu}_{0} c}{2 \pi a}\right)^{1 / 2}, \quad \epsilon_{0}=\frac{24 n}{\left(n^{2}+2\right)^{2}} k_{0},
$$

(the last equation takes into account that $k_{\nu} \ll n$ in this limit). Substituting the above equations for $\epsilon_{\nu}^{\text {abs }}$ and $\epsilon_{\nu}^{\mathrm{em}}$ into Equation B1, we obtain

$$
T_{\mathrm{g}}=\left[\frac{21}{640 \pi^{3}} \frac{L_{\mathrm{NS}}}{\sigma_{\mathrm{SB}} r^{2} \epsilon_{0}}\left(\frac{h \nu_{0}}{k}\right)^{2}\right]^{1 / 6}=\left(\frac{21}{640 \pi^{3}} \frac{L_{\mathrm{NS}}}{\sigma_{\mathrm{SB}} \epsilon_{0} r^{2}} \frac{h^{2} c \tilde{\nu}_{0}}{2 \pi k^{2} a}\right)^{1 / 6},
$$

where $\sigma_{\mathrm{SB}}$ is the Stefan Boltzmann constant, $L_{\mathrm{NS}}=4 \pi R_{\mathrm{NS}}^{2} \sigma_{\mathrm{SB}} T_{\mathrm{NS}}^{4}$ is the star's bolometric luminosity. These equations are applicable when three conditions are fulfilled: $\epsilon_{\nu}<1, \nu<\tilde{\nu}_{0}$, and $\nu<c / 2 \pi a$, where $\nu \sim 3 k T / h$. The quantities $\epsilon_{0}$ and $\nu_{0}$ in Equation (B6) can be evaluated from measurements of optical properties of grain material for a chosen $\tilde{\nu}_{0}$. Using measurements for amorphous magnesium silicate dust grains as listed in the Jena Database of Optical Constants for Cosmic Dust, we obtain $\epsilon_{0}=0.36$ for $\tilde{\nu}_{0}=6 \times 10^{12} \mathrm{~Hz}$ (corresponding to the Herschel red band wavelength of $160 \mu \mathrm{m})$. This gives the following estimate for the temperature at distance $r$ :

$$
T_{\mathrm{g}}=39\left(\frac{L_{30}}{r_{14}^{2} a}\right)^{1 / 6} \mathrm{~K},
$$

where $L_{30}$ is the bolometric NS luminosity in units of $10^{30} \mathrm{erg} \mathrm{s}^{-1}, a$ is the grain radius in $\mu \mathrm{m}$, and $r_{14}$ is the distance from the NS in units of $10^{14} \mathrm{~cm}$. This equation is valid for $T<\min \left(96 \mathrm{~K}, 2300 a_{\mu \mathrm{m}}^{-1} \mathrm{~K}\right)$. 